\title{
Isaac Newton
}

Mathématicien, Physicien, Philosophe anglais

(1643-1727)

\section{Principes mathématiques de la Philosophie naturelle}

(Philosophiae Naturalis Principia Mathematica)

Par feue Madame la Marquise du Chastellet

Paris, 1759

\section{Livre troisième. Du Système du Monde. (Début) Règles qu'il faut suivre dans l'étude de la Physique. Phénomènes. Propositions.}

Un document produit en version numérique par Jean-Marc Simonet, bénévole,
Courriel : Jean-Marc Simonet@uqac.ca

Dans le cadre de la collection: "Les classiques des sciences sociales"

Site web : http://classiques.uqac.ca/

Une collection développée en collaboration avec la Bibliothèque

Paul-Émile-Boulet de l'Université du Québec à Chicoutimi

Site web : http://bibliotheque.uqac.ca/ 


\section{Politique d'utilisation de la bibliothèque des Classiques}

Toute reproduction et rediffusion de nos fichiers est interdite, même avec la mention de leur provenance, sans l'autorisation formelle, écrite, du fondateur des Classiques des sciences sociales, Jean-Marie Tremblay, sociologue.

Les fichiers des Classiques des sciences sociales ne peuvent sans autorisation formelle:

- être hébergés (en fichier ou page web, en totalité ou en partie) sur un serveur autre que celui des Classiques.

- servir de base de travail à un autre fichier modifié ensuite par tout autre moyen (couleur, police, mise en page, extraits, support, etc...),

Les fichiers (.html, .doc, .pdf, .rtf, .jpg, .gif) disponibles sur le site Les Classiques des sciences sociales sont la propriété des Classiques des sciences sociales, un organisme à but non lucratif composé exclusivement de bénévoles.

Ils sont disponibles pour une utilisation intellectuelle et personnelle et, en aucun cas, commerciale. Toute utilisation à des fins commerciales des fichiers sur ce site est strictement interdite et toute rediffusion est également strictement interdite.

\section{L'accès à notre travail est libre et gratuit à tous les utilisa- teurs. C'est notre mission.}

Jean-Marie Tremblay, sociologue

Fondateur et Président-directeur général, LES CLASSIQUES DES SCIENCES SOCIALES. 
Cette édition électronique a été réalisée par Jean-Marc Simonet, ancien professeur des Universités, bénévole.

Courriel : Jean-Marc Simonet@uqac.ca

À partir de :

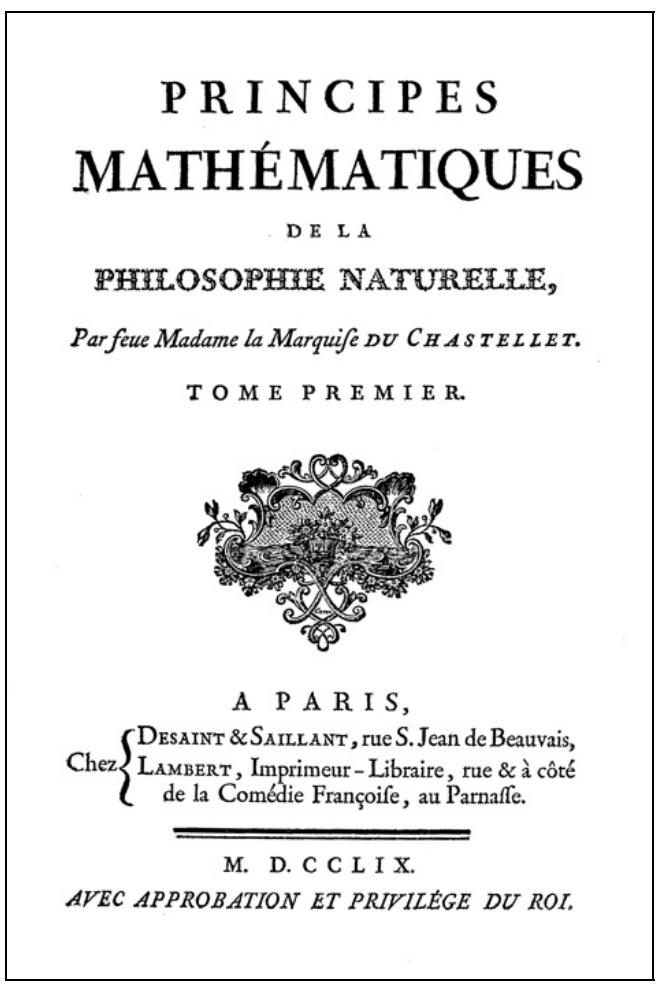

\section{Isaac Newton}

Physicien, Mathématicien, Philosophe anglais

$(1643-1727)$

\section{Principes mathématiques de la Philosophie naturelle}

Par feue Madame la marquise du Chastellet

chez Desaint \& Saillant et Lambert, imprimeurs, Paris, 1759 , deux tomes de 437 p. et 379 p.

Polices de caractères utilisées :

Pour le texte: Times New Roman, 14 et 12 points.

Pour les notes de bas de page : Times New Roman, 10 points.

Édition électronique réalisée avec le traitement de textes Microsoft Word 2008 pour Macintosh.

Mise en page sur papier format : LETTRE (US letter), 8.5' $\mathrm{x} 11{ }^{\prime \prime}$ )

Édition numérique réalisée le 3 juin 2010 à Chicoutimi, Ville de Saguenay, province de Québec, Canada

\section{Fait avec}




\section{Table des matières}

\section{Principes mathématiques de la Philosophie Naturelle.}

Du Système du Monde - Livre Troisième.

Règles qu'il faut suivre dans l'étude de la physique.

Phénomènes.

Propositions.

Du mouvement des nœuds de la Lune. 


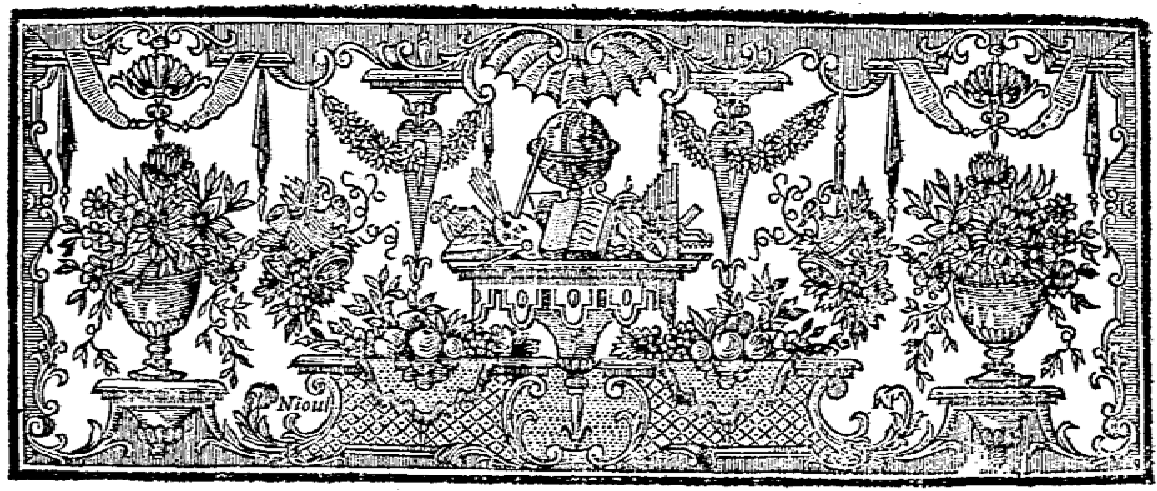

Table des matières

\section{DU SYSTÈME DU MONDE}

\section{Livre Troisième}

J'ai donné dans les Livres précédents les principes de la Philosophie naturelle, et je les ai traités plutôt en Mathématicien qu'en Physicien, car les vérités mathématiques peuvent servir de base à plusieurs recherches philosophiques, telles que les lois du mouvement et des forces motrices. Et afin de rendre les matières plus intéressantes, j'y ai joint quelques scholies dans lesquels j'ai traité de la densité des corps et de leur résistance, du vide, du mouvement du son et de celui de la lumière ; qui sont à proprement parler, des recherches plus physiques. Il me reste à expliquer par les mêmes principes mathématiques le système général du Monde.

J'avais d'abord traité l'objet de ce troisième Livre par une Méthode moins mathématique, afin qu'il puisse être à la portée de plus de personnes. Mais de crainte de donner lieu aux chicanes de ceux qui ne voudraient pas quitter leurs anciens préjugés, parce qu'ils ne sentiraient pas la force des conséquences que je tire de mes principes, faute d'avoir assez médité les Propositions que j'ai données dans les Livres précédents, j’ai rédigé ce Livre en plusieurs Propositions, selon la méthode des Mathématiciens, pour ceux qui auront lu les deux premiers 
Livres, car c'est pour eux que ce troisième Livre est destiné, et comme il y a dans les deux premiers Livres plusieurs Propositions qui pourraient arrêter longtemps, même les Mathématiciens, je ne prétends pas exiger qu'ils lisent ces deux premiers Livres entiers; il leur suffira d'avoir lu attentivement les Définitions, les Lois du Mouvement, et les trois premières Sections du premier Livre, et ils pourront passer ensuite ce troisième Livre, qui traite du Système du Monde, et avoir soin seulement de consulter les autres Propositions des deux premiers Livres lorsqu'ils les trouveront citées et qu'ils en auront besoin. 


\section{RÈGLES QU'IL FAUT SUIVRE DANS L'ÉTUDE DE LA PHYSIQUE}

\section{RĖGLE PREMIÈRE.}

Il ne faut admettre de causes, que celles qui sont nécessaires pour expliquer les Phénomènes.

La nature ne fait rien en vain, et ce serait faire des choses inutiles que d'opérer par un plus grand nombre de causes ce qui peut se faire par un plus petit.

\section{RÈGLE II.}

Les effets du même genre doivent toujours être attribués, autant qu'il est possible, à la même cause.

Ainsi la respiration de l'homme et celle des bêtes; la chute d'une pierre en Europe et en Amérique ; la lumière du feu d'ici-bas et celle du Soleil ; la réflexion de la lumière sur la Terre et dans les Planètes, doivent être attribuées respectivement aux mêmes causes.

\section{RÈGLE III.}

Les qualités des corps qui ne sont susceptibles ni d'augmentation ni diminution, et qui appartiennent à tous les corps sur lesquels on peut faire des expériences, doivent être regardées comme appartenant à tous les corps en général.

On ne peut connaître les qualités des corps que par l'expérience, ainsi on doit regarder comme des qualités générales celles qui se trouvent dans tous les corps, et qui ne peuvent souffrir de diminution, car il est impossible de dépouiller les corps des qualités qu'on ne peut diminuer. On ne peut pas opposer des rêveries aux expériences, et on ne 
doit point abandonner l'analogie de la nature qui est toujours simple et semblable à elle-même.

L'étendue des corps ne se connaît que par les sens, et elle ne se fait pas sentir dans tous les corps : mais comme l'étendue appartient à tous ceux qui tombent sous nos sens, nous affirmons qu'elle appartient à tous les corps en général.

Nous éprouvons que plusieurs corps sont durs : or la dureté du tout vient de la dureté des parties, ainsi nous admettons cette qualité non seulement dans les corps dans lesquels nos sens nous la font éprouver, mais nous en inférons, avec raison, que les particules indivisées de tous les corps doivent être dures.

Nous concluons de la même manière, que tous les corps sont impénétrables. Car tous ceux que nous touchons étant impénétrables, nous regardons l'impénétrabilité comme une propriété qui appartient à tous les corps.

Tous les corps que nous connaissons étant mobiles, et doués d'une certaine force (que nous appelions force d'inertie) par laquelle ils persévèrent dans le mouvement ou dans le repos, nous concluons que tous les corps en général ont ces propriétés. L'extension, la dureté, l'impénétrabilité, la mobilité, et l'inertie du tout vient donc de l'extension, de la dureté, de l'impénétrabilité, de la mobilité, et de l'inertie des parties : d'où nous concluons que toutes les petites parties de tous les corps sont étendues, dures, impénétrables, mobiles, et douées de la force d'inertie. Et c'est là le fondement de toute la Physique.

De plus, nous savons encore par les phénomènes, que les parties contiguës des corps peuvent se séparer, et les Mathématiques font voir que les parties indivisées les plus petites peuvent être distinguées l'une de l'autre par l'esprit. On ignore encore si ces parties distinctes et non divisées, pourraient être séparées par les forces de la nature, mais s'il était certain, par une seule expérience, qu'une des parties, qu'on regarde comme indivisibles, eût souffert quelque division en séparant ou brisant un corps dur quelconque : nous conclurions par cette règle, que non seulement les parties divisées sont séparables, mais que celles qui sont indivisées peuvent se diviser à l'infini. 
Enfin, puisqu'il est constant par les expériences et par les observations astronomiques, que tous les corps qui sont près de la surface de la Terre pèsent sur la Terre, selon la quantité de leur matière; que la Lune pèse sur la Terre à raison de sa quantité de matière, que notre mer pèse à son tour sur la Lune, que toutes les planètes pèsent mutuellement les unes sur les autres, et que les comètes pèsent aussi sur le Soleil, on peut conclure, suivant cette troisième règle que tous les corps gravitent mutuellement les uns vers les autres. Et ce raisonnement en faveur de la gravité universelle des corps, tiré des phénomènes, sera plus fort que celui par lequel on conclut leur impénétrabilité ; car nous n'avons aucune expérience ni aucune observation qui nous assure que les corps célestes sont impénétrables. Cependant je n'affirme point que la gravité soit essentielle aux corps. Et je n'entends par la force qui rende dans les corps, que la seule force d'inertie, laquelle est immuable ; au lieu que la gravité diminue lorsqu'on s'éloigne de la Terre.

\section{RÈGLE IV.}

Dans la Philosophie expérimentale, les proportions tirées par induction des phénomènes doivent être regardées malgré les hypothèses contraires, comme exactement ou à peu près vraies, jusqu'à ce que quelques autres phénomènes les confirment entièrement ou fassent voir qu'elles sont sujettes à des exceptions.

Car une hypothèse ne peut affaiblir les raisonnements fondés sur l'induction tirée de l'expérience. 


\section{PHÉNOMÈNES}

\section{PHÉNOMÈNE PREMIER.}

Les satellites de Jupiter décrivent autour de cette Planète des aires proportionnelles aux temps, et leurs temps périodiques (en supposant que les étoiles fixes soient en repos) sont en raison sesquiplée de leurs distances au centre de cette Planète.

C'est ce qui est constaté par les observations astronomiques. Car les orbes de ces planètes sont à peu près des cercles concentriques à Jupiter, et leurs mouvements dans ces cercles paraissent uniformes. À l'égard de leurs temps périodiques tous les Astronomes conviennent qu'ils sont en raison sesquiplée des demi-diamètres de leurs orbes, et c'est ce qu'on va voir par la table suivante.

Temps périodiques des fatellites de Jupiter.

$$
\begin{gathered}
\mathrm{I}^{\mathrm{J}} 18^{\mathrm{h}} 27^{\prime} 34^{\prime \prime} \cdot 3^{\mathrm{J}} 13^{\mathrm{h}} \mathrm{I} 3^{\prime} 4^{\prime \prime} \cdot 7^{\mathrm{J}} 3^{\mathrm{h}} 4^{\prime} 30^{\prime \prime} \cdot 16^{\mathrm{J}} 16^{\mathrm{h}} 32^{\prime} 9^{\prime \prime} . \\
\text { Diftances des Jatellites au centre de Jupiter. }
\end{gathered}
$$

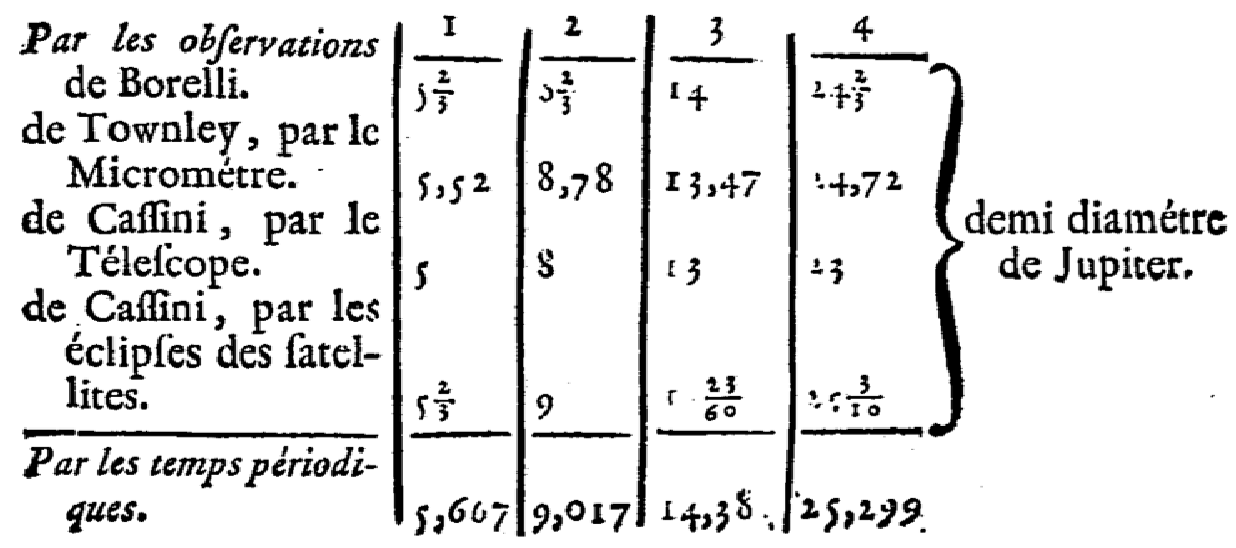

Les élongations des satellites de Jupiter et son diamètre ont été déterminés très exactement par le Docteur Pound avec d'excellents micromètres de la manière suivante.

La plus grande élongation héliocentrique du quatrième satellite au centre de Jupiter fut prise avec un micromètre placé dans un tube de 
15 pieds, et elle se trouva de $8^{\prime} 16^{\prime \prime}$ environ dans la moyenne distance de Jupiter à la Terre.

Celle du troisième satellite fut prise avec un télescope de 123 pieds armé d'un micromètre, et elle se trouva à la même distance de Jupiter à la Terre, de 4' 42". Les plus grandes élongations des autres satellites, à la même distance de Jupiter à la Terre, sont, par les temps périodiques, de 2' 56" 47"', et de 1'51" 6"'.

Le diamètre de Jupiter fut pris souvent avec un micromètre placé dans un télescope de 123 pieds, et ce diamètre étant réduit à la moyenne distance de Jupiter au Soleil ou à la Terre, il se trouva toujours avoir moins de 40", mais jamais moins que 38", et il en avait souvent 39". Avec des télescopes moins grands ce diamètre est de 40" ou de 41". Car la lumière de Jupiter à cause de l'inégale réfrangibilité des rayons, est un peu dilatée, et cette dilatation a une moindre raison au diamètre de Jupiter dans les grands télescopes qui sont faits avec exactitude, que dans ceux qui sont plus petits ou moins parfaits.

Dans les observations des passages du premier et du troisième satellite sur le disque de Jupiter, par lesquelles on détermina les temps écoulés depuis le commencement de l'entrée sur le disque jusqu'au commencement de la sortie, et depuis l'entrée totale jusqu'à la sortie totale, on employa un télescope de la même longueur. Et le diamètre de Jupiter dans sa moyenne distance à la Terre se trouva, par le passage du premier satellite, de $37 \frac{1}{8}$ ", et par le passage du troisième, de $37 \frac{3}{8}$ ". Mais le temps que l'ombre du premier satellite employa à traverser le disque de Jupiter ayant été observé, il donna le diamètre de Jupiter de 37" environ, dans la moyenne distance de Jupiter à la Terre. Prenant donc environ $37 \frac{1}{4}$ " pour ce diamètre, les plus grandes élongations du premier, du second, du troisième, et du quatrième satellite mesurées en demi-diamètres de Jupiter sont de 5,$965 ; 9,494 ; 15,141$; et 26,63 ; respectivement.

\section{PHÉNOMÈNE II.}

Les satellites de Saturne, décrivent autour de cette Planète des aires proportionnelles aux temps; et leurs temps périodiques, (les étoiles fixes étant supposées en repos) sont en raison sesquiplée de leurs distances au centre de Saturne. 
Les observations de Cassini donnent les distances de ces planètes au centre de Saturne, et leurs temps périodiques, tels qu'ils sont marqués dans la table suivante.

Temps périodiques des fatellites de Saturne.

$8^{\mathrm{J}} 21^{\mathrm{h}} 1^{\prime}{ }^{\prime} 7^{\prime \prime} \cdot 2^{\mathrm{J}} 17^{\mathrm{h}} 41^{\prime} 22^{\prime \prime} \cdot 4^{\mathrm{J}} 12^{\mathrm{h}} 25^{\prime} \mathrm{I} 2^{\prime \prime} \cdot 15^{\mathrm{J}} 22^{\mathrm{h}} 41^{\prime} 14^{\prime \prime}$.

$79^{\mathrm{J}} 7^{\mathrm{h}} 48^{\prime} \circ 9^{\prime \prime}$.

Diftances des fatellites au centre de Saturne en demi diamétres de Son anneau.

Par les obfervations. $\quad x \frac{19}{20} . \quad 2 \frac{1}{2} . \quad 3 \frac{2}{2} . \quad 8.24$. Par les temps périodiques. $1,93 \cdot 2,47 \cdot 3 \cdot 45 \cdot 8.23,35$.

Les observations donnent ordinairement pour la plus grande élongation du quatrième satellite au centre de Saturne environ huit demidiamètres. Mais cette plus grande élongation prise avec un excellent micromètre adapté à un télescope d'Huygens de 123 pieds, a été trouvée de huit demi-diamètres et $\frac{7}{10}$. Par cette observation et par les temps périodiques, les distances des satellites au centre de Saturne sont en demi-diamètres de son anneau de 2,$1 ; 2,69 ; 3,75 ; 8,7$; et 25,35 .

Le diamètre de Saturne, par le même télescope, était au diamètre de son anneau, comme 3 à 7, et le diamètre de l'anneau les 28 et 29 mai de l'année 1719 fut trouvé de 43", ce qui donne 42" pour le diamètre de l'anneau dans la moyenne distance de Saturne à la Terre, et 18 " pour le diamètre de Saturne. C'est ainsi qu'on les trouve avec les meilleurs et les plus grands télescopes, car dans les grands télescopes, les grandeurs apparentes des corps célestes ont une plus grande proportion à la dilatation de la lumière vers les bords de leurs disques, que dans les petits. Si on ôte toute la lumière erratique, le diamètre de Saturne sera à peine de 16".

\section{PHÉNOMÈNE III.}

Les cinq principales planètes, Mercure, Vénus, Mars, Jupiter et Saturne enferment le Soleil dans leurs orbes.

Il est prouvé par les phases de Mercure et de Vénus que ces planètes tournent autour du Soleil. Lorsque tout leur disque est éclairé elles 
sont au-delà du Soleil, quand leur disque est à moitié obscurci elles sont en quadrature avec le Soleil ; et quand elles paraissent en croissant elles sont entre le Soleil et nous, et quelquefois elles passent sur son disque sur lequel elles paraissent alors comme des espèces de taches. On est certain que Mars enferme le Soleil dans son orbe, parce que son disque est entièrement éclairé lorsqu'il est prêt d'être en conjonction avec le Soleil, et qu'il est gibbeux dans ses quadratures. La même chose est prouvée pour Saturne et pour Jupiter parce qu'ils nous paraissent toujours entièrement éclairés : et la projection des ombres de leurs satellites sur leur globe prouve que ces planètes empruntent leur lumière du Soleil.

\section{PHÉNOMÈNE IV.}

Les temps périodiques des cinq principales planètes autour du Soleil, et celui de la Terre autour du Soleil ou du Soleil autour de la Terre, (en supposant les étoiles fixes en repos) sont en raison sesquiplée de leur moyenne distance au Soleil.

Tout le monde sait que cette Proportion a été découverte par Kepler. Les temps périodiques et les dimensions des orbites sont les mêmes, soit que le Soleil tourne autour de la Terre, soit que la Terre tourne autour du Soleil. Tous les Astronomes conviennent de la raison dans laquelle sont les temps périodiques. Mais pour les grandeurs des orbites, Kepler et Bouillaut sont ceux qui les ont déterminées avec le plus de soin d'après les observations : et les distances moyennes, qui répondent aux temps périodiques, ne diffèrent pas sensiblement des distances qu'ils ont trouvées, et elles sont pour la plûpart moyennes entre ce que donnent leurs observations, comme on peut le voir dans la table suivante. 
Temps périodiques de la terre \& des planettes autour du Soleil par. rapport aux fixes, en jours \& en parties décimales de jour.

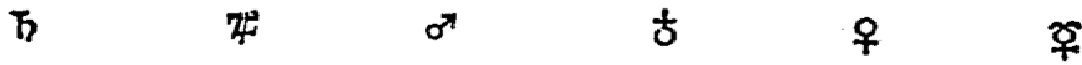
10759,275 . 4332,514. 686,9785. 365,2565. 224,6176. 87,9692.

Diffances moyennes des planettes \& de la terre au Soleil.

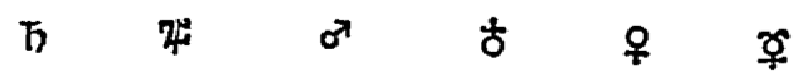

Selon Kepler. $\quad$ 951000. 519650. I 52350.100000 .72400 .38806$.

Selon Bouillaut. 954198.522520 .152350 .100000 .72398 .38585 .

Selon les temps

périodiques. 954006.520096. I52369.100000.72333.38710.

Il n'y a point de disputes sur les distances de Vénus et de Mercure au Soleil, car elles sont déterminées par leurs élongations au Soleil. Et les éclipses des satellites de Jupiter ôtent toute espèce de doute sur les distances au Soleil des planètes supérieures. Car par ces éclipses on détermine la position de l'ombre que Jupiter projette, et par-là, on a la longitude héliocentrique de Jupiter. Et les longitudes héliocentriques et géocentriques comparées entre elles déterminent la distance de Jupiter.

\section{PHÉNOMÈNE V.}

Si on prend la Terre pour centre des révolutions des planètes principales, les aires qu'elles décrivent ne seront point proportionnelles aux temps; mais si on regarde le Soleil comme le centre de leurs mouvements, on trouvera alors leurs aires proportionnelles aux temps.

Dans la première de ces suppositions on trouverait que les planètes avancent quelquefois, que quelquefois elles sont stationnaires, et que d'autres fois elles sont rétrogrades : mais dans la seconde elles avancent toujours, et cela d'un mouvement à peu près uniforme, qui est cependant un peu plus prompt dans leurs périhélies, et plus lent dans leurs aphélies, de sorte que les aires sont toujours égales en temps égaux. Cette Proposition est très connue des Astronomes, et elle est démontrée surtout avec une grande évidence pour la planète de Jupiter par les éclipses de ses satellites, lesquelles, comme nous avons déjà 
dit, déterminent les longitudes héliocentriques de cette planète et ses distances au Soleil.

\section{PHÉNOMÈNE VI.}

La Lune décrit autour de la Terre des aires proportionnelles aux temps.

Cela se prouve par le mouvement angulaire de la Lune, et par son diamètre apparent. Les mouvements de la Lune sont à la vérité un peu troublés par la force du Soleil, mais je néglige dans ces Phénomènes ces petites erreurs insensibles. 


\section{PROPOSITIONS}

\section{Proposition I. - THÉORÈME I.}

Les forces par lesquelles les satellites de Jupiter sont retirés perpétuellement du mouvement rectiligne et retenus dans leurs orbites, tendent au centre de Jupiter et sont en raison réciproque des carrés de leurs distances à ce centre.

La première partie de cette Proposition est prouvée par le Phénomène 1 et par la seconde et la troisième Proposition du premier Livre : et la dernière l'est par le premier Phénomène, et par le Cor. 6 de la Prop. 4 du même Livre.

Il en est de même des satellites de Saturne par le Phénomène 2.

\section{Proposition II. - THÉORÈME II.}

Les forces par lesquelles les planètes principales sont perpétuellement retirées du mouvement rectiligne, et retenues dans leurs orbites, tendent au Soleil, et sont réciproquement comme le carré de leurs distances à son centre.

La première partie de cette Proposition se prouve par le Phénomène 5 et par la seconde Proposition du Livre 1. l'autre partie se prouve par le Phénomène 4 et la Prop. 4 du même Livre. Cette seconde partie de la Proposition se démontrerait encore très rigoureusement par la fixité des aphélies. Car pour peu que les planètes s'écartassent de cette loi le mouvement des apsides serait remarquable à chaque révolution, (par le Cor. 1 de la Prop. 45 Liv. 1) et deviendrait très considérable au bout de plusieurs révolutions.

\section{Proposition III. - THÉORÈME III.}

La force qui retient la Lune dans son orbite, tend vers la Terre, et est en raison réciproque du carré de la distance des lieux de la Lune au centre de la Terre. 
La première partie de cette Proposition se prouve par le Phénomène 6 et par les Propositions 2 et 3 du premier Livre, et la dernière par le mouvement très lent de l'apogée lunaire. Car ce mouvement, qui à chaque révolution n'est que de trois degrés et de trois minutes en conséquence, peut être négligé. Or il est clair (par le Cor. 1 de la Prop. 45 Liv. 1) que si on prend le rapport de $D$ à 1 pour exprimer celui de la distance de la Lune du centre de la Terre au demi-diamètre de la Terre ; la force qui produit ce mouvement, sera réciproquement comme $D^{2} \frac{4}{243}$, c'est-à-dire, en une raison un peu plus grande que la raison doublée inverse de la distance, mais qui approche plus de $59 \frac{3}{4}$ parties de la doublée que de la triplée ; et comme la différence de cette force à celle qui serait exactement en raison inverse du carré, vient de l'action du Soleil, (comme je l'expliquerai dans la suite) on peut la négliger ici. L'action du Soleil en tant qu'il détourne la Lune de la Terre, est à peu près comme la distance de la Lune à la Terre; donc (par ce qui a été dit dans le Cor. 2 de la Prop. 45 du Liv. 1) elle est à la force centripète de la Lune comme 2 à 357,45 à peu près, ou comme 1 à $178 \frac{29}{40}$. Et en négligeant cette petite action du Soleil, la force restante par laquelle la Lune est retenue dans son orbite, sera réciproquement comme $D^{2}$, ce qui paraîtra clairement en comparant cette force avec la force de la gravité, comme dans la Proposition suivante.

Cor. Si la force centripète médiocre par laquelle la Lune est retenue dans son orbite est premièrement augmentée dans la raison de $177 \frac{29}{40}$, à $178 \frac{29}{40}$, et ensuite en raison doublée du demi-diamètre de la Terre à la moyenne distance du centre de la Lune au centre de la Terre : on aura la force centripète de la Lune près de la surface de la Terre, en supposant que cette force, en descendant vers la surface de la Terre, augmente continuellement en raison doublée inverse de la hauteur.

\section{Proposition IV. - THÉORÈME IV.}

La Lune gravite vers la Terre, et par la force de la gravité elle est continuellement retirée du mouvement rectiligne et retenue dans son orbite.

La moyenne distance de la Lune à la Terre dans les syzygies est, suivant Ptolomée et plusieurs Astronomes, de 59 demi-diamètres de la 
Terre, Vendelinus et Huygens la font de 60, Copernic de $60 \frac{1}{3}$, Street de $60 \frac{2}{5}$ et Tycho de $56 \frac{1}{2}$. Mais Tycho et tous ceux qui suivent ses tables de réfraction, supposent que les réfractions du Soleil et de la Lune sont plus grandes que celles des étoiles fixes, de 4 ou 5 minutes environ, (ce qui est entièrement contraire à ce qu'on connaît de la lumière) et par-là ils ont augmenté la parallaxe de la Lune d'autant de minutes, c'est-à-dire, presque de la douzième ou de la quinzième partie de toute sa parallaxe.

En corrigeant cette erreur, on trouvera cette distance déterminée par Tycho de $60 \frac{1}{2}$ demi-diamètres de la Terre environ, c'est-à-dire, telle à peu près que les autres Astronomes l'avaient trouvée.

Prenons 60 demi-diamètres de la Terre pour la distance moyenne dans les syzygies; et supposons que la révolution de la Lune autour de la Terre, par rapport aux étoiles fixes, s'achève en 27 jours 7 heures 43 minutes, comme les Astronomes l'ont déterminé : enfin prenons 123249600 pieds de Paris pour la circonférence de la Terre, suivant les mesures prises en France : on aura $15 \frac{1}{2}$ pieds de Paris pour l'espace que la Lune parcourait en une minute, si elle était privée de tout autre mouvement et qu'elle descendit vers la Terre par la seule force qui la retient (selon le Cor. de la Prop. 3) dans son orbite : ce qui est aisé à tirer, par le calcul, soit de la Prop. 36 du Liv. 1 ou (ce qui revient au même) du Cor. 9 de la quatrième Proposition du même Livre. Car le sinus verse de l'arc que la Lune parcourt en une minute, dans son mouvement moyen, à la distance de 60 demi-diamètres de la Terre, est de $15 \frac{1}{12}$ pieds de Paris environ, ou plus exactement de 15 pieds un pouce et $1 \frac{4}{9}$ lignes. Or, comme cette force doit augmenter en approchant de la Terre en raison doublée inverse de la distance, et que par conséquent elle doit être $60 \times 60$ fois plus grande à la surface de la Terre qu'à la distance où est la Lune ; un corps qui tomberait avec cette force, devrait parcourir ici-bas dans une minute $60 \times 60 \times 15 \frac{1}{12}$ pieds de Paris, et dans une seconde $15 \frac{1}{12}$ pieds de Paris ou plus exactement 15 pieds 1 pouce et $1 \frac{4}{9}$ lignes. Et c'est en effet l'espace que les corps décrivent dans une seconde en tombant vers la Terre. Car la longueur du pendule qui bat les secondes dans la latitude de Paris, est de 3 pieds de Paris et 8 lignes et demie, selon que M. Huygens l'a déterminé ; et la hauteur qu'un corps grave parcourt en tombant pendant 
une seconde, est à la demi longueur de ce pendule en raison doublée de la circonférence du cercle à son diamètre (comme M. Huygens l'a aussi déterminé) c'est-à-dire, que cette hauteur est de 15 pieds de $\mathrm{Pa}$ ris 1 pouce et $1 \frac{7}{9}$ lignes. Donc la force par laquelle la Lune est retenue dans son orbite, serait égale à la force de la gravité ici-bas, si la Lune était près de la surface de la Terre, donc (selon les Règles 1 et 2) c'est cette même force que nous appelons gravité. Car si cette force était autre que la gravité, les corps en s'approchant de la Terre par ces deux forces réunies descendraient deux fois plus vite, et ils parcouraient en tombant pendant une seconde un espace de $30 \frac{1}{6}$ pieds de Paris : ce qui est entièrement contraire à l'expérience.

Ce calcul est fondé sur l'hypothèse que la Terre soit en repos, car si la Terre et la Lune se meuvent autour du Soleil, et qu'elles tournent en même temps autour de leur commun centre de gravité : la distance respective des centres de la Lune et de la Terre sera de $60 \frac{1}{2}$ demidiamètres de la Terre environ, la loi de la gravité demeurant la même ; c'est ce qu'on verra clairement si on veut faire le calcul, lequel ne demande que la Prop. 60 du Livre 1.

\section{SCHOLIE.}

On peut rendre la démonstration de cette Proposition plus sensible, par le raisonnement suivant. Si plusieurs Lunes faisaient leurs révolutions autour de la Terre, ainsi que dans le système de Jupiter ou de Saturne, leurs temps périodiques, par l'induction, suivraient la loi découverte par Kepler, et par conséquent leurs forces centripètes (Prop. 1 de ce Livre) seraient réciproquement comme les carrés de leurs distances au centre de la Terre. Et si celle de ces Lunes qui serait la plus proche de la Terre était petite, et qu'elle touchât presque le sommet des plus hautes montagnes : la force centripète, par laquelle cette Lune serait retenue dans son orbite, serait, suivant le calcul précédent, à peu près égale à celle des corps graves placés sur le sommet de ces montagnes. De sorte que si cette même petite Lune était privée de tout le mouvement par lequel elle avance dans son orbe, et qu'elle n'eût plus par conséquent de force centrifuge, elle descendrait vers la Terre avec la même vitesse que les corps graves placés au sommet de ces montagnes tombent vers la Terre, et cela à cause de l'égalité qui serait entre 
la gravité et la force qui agirait alors sur cette petite Lune. Or si la force par laquelle cette petite Lune descend était autre que la gravité, et que cependant elle pesât sur la Terre comme les corps graves placés au sommet de ces montagnes, cette petite Lune devrait par ces deux forces réunies descendre deux fois plus vite. Donc, puisque ces deux forces, c'est-à-dire, celles des corps graves et celles de ces petites Lunes, sont dirigées vers le centre de la Terre, et qu'elles sont égales et semblables entre elles, ces forces sont les mêmes et par conséquent elles doivent avoir (Règles 1 et 2) une même cause. Donc la force, qui retient la Lune dans son orbite, est celle-là même que nous appelons gravité : puisque sans cela cette petite Lune n'aurait point de gravité au sommet de cette montagne, ou bien elle tomberait deux fois plus vite que les graves.

\section{Proposition V. - THÉORÈME V.}

Les satellites de Jupiter gravitent vers Jupiter, ceux de Saturne vers Saturne, et les planètes principales vers le Soleil, et c'est par la force de leur gravité que ces corps révolvants sont retirés à tout moment de la ligne droite et qu'ils sont retenus dans des orbites curvilignes.

Car les révolutions des satellites de Jupiter autour de Jupiter, celles des satellites de Saturne autour de Saturne, et celles de Mercure, de Vénus et des autres planètes principales autour du Soleil, sont des Phénomènes du même genre que celui de la révolution de la Lune autour de la Terre ; et par conséquent, par la seconde Règle, ils doivent dépendre de causes du même genre : surtout puisqu'il est démontré, que les forces dont dépendent ces révolutions tendent au centre de Jupiter, de Saturne et du Soleil, et qu'en s'éloignant de Jupiter, de Saturne et du Soleil, ces forces décroissent dans la même raison, dans laquelle la force de la gravité décroît en s'éloignant de la Terre.

Cor. 1. Toutes les planètes sont donc pesantes. Car personne ne doute que Vénus, Mercure et toutes les autres planètes ne soient des corps du même genre que Jupiter et Saturne. Et comme toute attraction est mutuelle par la troisième loi du mouvement, Jupiter doit graviter vers tous ses satellites, Saturne vers tous les siens, la Terre vers la Lune, et le Soleil vers toutes les planètes principales. 
Cor. 2. La gravité vers chaque planète est réciproquement comme le carré de la distance à son centre.

Cor. 3. Par les Cor. 1 et 2 toutes les planètes gravitent les unes vers les autres, ainsi Jupiter et Saturne en s'attirant mutuellement, troublent sensiblement leurs mouvements vers leur conjonction, le Soleil trouble ceux de la Lune, et le Soleil et la Lune ceux de notre mer, comme je l'expliquerai dans la suite.

\section{SCHOLIE.}

Nous avons appelé jusqu'ici la force qui retient les corps célestes dans leur orbite force centripète. On a prouvé que cette force est la même que la gravité, ainsi dans la suite nous l'appellerons gravité. Car la cause de cette force centripète, qui retient la Lune dans son orbite, doit s'étendre à toutes les planètes par les Règles 1,2 et 4 .

\section{Proposition VI. — THÉORÈME VI.}

Tous les corps gravitent vers chaque planète, et sur la même planète quelconque leurs poids, à égale distance du centre, sont proportionnels à la quantité de matière que chacun d'eux contient.

Tous les corps descendent vers la Terre dans des temps égaux (en faisant abstraction de l'inégale retardation causée par la petite résistance de l'air) c'est ce que plusieurs Philosophes avaient déjà observé, et ce qu'on peut connaître avec précision par l'égalité des temps dans lesquels se font les oscillations des pendules. J'en ai fait l'expérience avec des pendules d'or, d'argent, de plomb, de verre, de sable, de sel commun, de bois, d'eau, et de froment. Pour y réussir, je fis faire deux boîtes de bois rondes et égales, j'en emplis une de bois, et je mis un poids égal d'or dans l'autre, en le plaçant aussi exactement que je le pus dans le point qui répondait au centre d'oscillation de la première boîte. Ces boîtes étaient suspendues à deux fils égaux de 11 pieds chacun, ainsi j'avais par-là deux pendules entièrement pareils quant au poids, à la figure, et à la résistance de l'air. Ces pendules, dont les poids étaient placés à côté l'un de l'autre firent des oscillations qui se suivirent pendant un très long temps. Donc, la quantité de matière de 
l'or, était à la quantité de matière du bois (par les Cor. 1 et 6 de la Prop. 24 du Liv. 2) comme l'action de la force motrice sur tout l'or à cette même action sur tout le bois, c'est-à-dire, comme le poids au poids. Il en fut de même dans les autres pendules. Dans ces expériences une différence d'un millième dans la matière des corps de même poids était aisée à apercevoir.

Il n'y a donc aucun doute que la nature de la gravité ne soit la même dans les planètes et sur la Terre. Car supposé que quelque corps terrestre fut élevé jusqu'à l'orbe de la lune, et que la lune et ce corps, étant privés de tout mouvement, fussent abandonnés à leur gravité, et tombassent ensemble vers la Terre ; il est certain, par ce qu'on a déjà dit, que ce corps et la lune parcouraient des espaces égaux en temps égaux, et que par conséquent son poids serait à celui de la lune en même raison que leurs quantités de matière.

De plus, comme les satellites de Jupiter font leurs révolutions autour de cette planète dans des temps qui sont en raison sesquiplée de leurs distances à son centre, leurs gravités accélératrices vers Jupiter seront réciproquement comme le carré de leurs distances à son centre, et par conséquent, à égales distances de Jupiter, elles seront égales. Ainsi ils parcourraient des espaces égaux en temps égaux en tombant vers Jupiter de hauteurs égales, comme il arrive aux graves sur notre Terre. Et par le même raisonnement les planètes qui tournent autour du Soleil, étant abandonnées à la force qui les porte vers cet astre, parcouraient en descendant vers lui des espaces égaux en temps égaux s'ils tombaient de hauteurs égales. Or les forces qui accélèrent également des corps inégaux sont comme ces corps, c'est-à-dire, que les poids des corps sur les planètes sont comme la quantité de matière qu'ils contiennent.

De plus, les poids de Jupiter et de ses satellites sur le Soleil sont proportionnels à leur quantité de matière, c'est ce qui est prouvé (Cor. 3 Prop. 65 Liv. 1) par le mouvement très régulier des satellites de Jupiter ; car si l'un de ces satellites était plus attiré que les autres vers le Soleil, parce qu'il contient plus de matière, le mouvement des satellites (Cor. 2 Prop. 65 Liv. 1) serait dérangé par cette inégale attraction. $\mathrm{Si}$, à distance égale du Soleil, un de ces satellites était plus pesant sur le Soleil à raison de la quantité de matière que Jupiter à raison de la sienne, dans une raison quelconque donnée, comme, par exemple, 
dans la raison de $d$ à $e$, la distance entre le centre du Soleil et le centre de l'orbe de ce satellite serait toujours plus grande que la distance entre le centre du Soleil et le centre de Jupiter à peu près en raison sousdoublée, comme je l'ai trouvé en faisant le calcul. Et si le satellite était moins pesant vers le Soleil dans cette raison de $d$ à $e$, la distance du centre de l'orbe du satellite au centre du Soleil serait moindre que la distance du centre de Jupiter au centre du Soleil dans cette même raison sous-doublée. Donc, si, à distances égales du Soleil, la gravité accélératrice d'un satellite quelconque vers le Soleil était plus grande ou plus petite que la gravité accélératrice de Jupiter vers le Soleil, seulement de la millième partie de la gravité totale; la distance du centre de l'orbe du satellite au Soleil serait plus ou moins grande que la distance de Jupiter au Soleil de $\frac{1}{2000}$ partie de la distance totale, c'est-àdire, de la cinquième partie de la distance du satellite le plus éloigné du centre de Jupiter, ce qui rendrait cet orbe très sensiblement excentrique. Mais les orbes des satellites sont concentriques à Jupiter, ainsi les gravités accélératrices de Jupiter et de ses satellites vers le Soleil sont égales entre elles. Par le même raisonnement, les poids de Saturne et de ses satellites sur le Soleil sont à des distances égales du Soleil, comme la quantité de matière que chacun d'eux contient : et la lune et la Terre ou ne pèsent point sur le Soleil, ou bien pèsent dans la proportion exacte de leurs masses : or par les Cor. 1 et 3 de la Prop. 5 on voit qu'ils doivent peser.

Ainsi les poids de chacune des parties d'une planète quelconque sur une autre planète sont entre eux comme la quantité de matière que chacune de ces parties contient. Car si quelques unes de ces parties gravitaient plus et d'autres moins que selon leur quantité de matière ; la planète totale graviterait dans une raison plus ou moins grande que celle de sa quantité de matière, suivant la nature des parties dont elle contiendrait une plus grande quantité ; et il n'importe que ces parties fussent extérieures ou intérieures à la planète. Qu'on suppose, par exemple, que les corps d'ici-bas soient élevés jusqu'à l'orbe de la Lune, et qu'on les compare avec le corps de la Lune: si leurs poids étaient aux poids des parties externes de la Lune comme les quantités de matière, et qu'ils fussent aux poids de ses parties internes dans une plus grande ou une moindre raison, ces mêmes corps seraient au poids de la Lune entière dans une plus grande ou une moindre raison : ce qui serait contraire à ce qu'on vient de prouver. 
Cor. 1. Ainsi, les poids des corps ne dépendent point de leur forme et de leur texture. Car si ces poids varient avec la forme, ils seraient tantôt plus grands, et tantôt moindres, selon les différentes formes, quoique la quantité de matière fut la même : ce qui est entièrement contraire à l'expérience.

Cor. 2. Tous les corps qui sont autour de la Terre pèsent sur la Terre, et leurs poids, lorsqu'ils sont également éloignés de son centre, sont comme la quantité de matière que chacun d'eux contient. C'est ce que les expériences ont fait voir dans tous les corps sur lesquels on a pu en faire. Ainsi, par la troisième règle, on doit affirmer la même chose de tous les corps en général. Si l'Ether ou quelque autre corps était entièrement privé de gravité, ou qu'il gravitât dans une moindre raison que celle de sa quantité de matière : comme cette espèce de corps ne serait différente des autres, suivant Aristote, Descartes et d'autres, que par la forme de ses parties, il pourrait arriver, que ces corps, en changeant peu à peu de forme, se changeraient dans l'espèce des corps qui gravitent en raison de leur quantité de matière; et au contraire les corps graves pourraient perdre par la suite des temps leur gravité en prenant la même forme que les premiers. Ainsi les poids dépendraient des formes et pourraient varier avec elles, contre ce qui a été prouvé dans le Cor. précédent.

Cor. 3. Tous les espaces ne sont pas également pleins. Car s'ils l'étaient, toute matière serait également dense, ainsi la gravité spécifique du fluide qui remplirait la région de l'air, ne céderait point à la gravité spécifique du vif argent, de l'or ou de quelque autre corps, quelque dense qu'il fut; ainsi l'or ni aucun autre corps quelconque ne pourrait descendre dans l'air. Car les corps ne descendent dans les fluides que parce qu'ils sont spécifiquement plus pesants. Or si la quantité de matière peut diminuer par la raréfaction jusqu'à un certain point dans un espace donné, pourquoi ne pourra-t-elle pas diminuer à l'infini ?

Cor. 4. Si les parties solides de tous les corps sont de la même densité, et qu'elles ne puissent se raréfier sans pores, il y a du vide. Je dis que les parties ont la même densité lorsque leurs forces d'inertie sont comme leur grandeur. 
Cor. 5. La force de la gravité est d'un autre genre que la force magnétique. Car l'attraction magnétique n'est point comme la quantité de matière attirée. Certains corps sont plus attirés par l'aimant, d'autres moins : et plusieurs ne le sont point du tout. La force magnétique d'un même corps peut être augmentée ou diminuée, elle est quelquefois beaucoup plus grande par rapport à la quantité de matière que la force de la gravité, elle ne décroît point en s'éloignant de l'aimant en raison doublée de la distance, mais presque en raison triplée, autant que j'ai pu le déterminer par des expériences assez grossières.

\section{Proposition VII. — THÉORÈME VII.}

La gravité appartient à tous les corps, et elle est proportionnelle à la quantité de matière que chaque corps contient.

On a prouvé ci-dessus que toutes les planètes gravitent mutuellement les unes vers les autres : que la gravité vers une planète quelconque, considérée à part, est réciproquement comme le carré de la distance au centre de cette planète : et que par conséquent (Prop. 69 Liv. 1 et les Cor.) la gravité dans toutes les planètes est proportionnelle à leur quantité de matière.

Mais comme toutes les parties d'une planète quelconque $A$, pèsent sur une autre planète quelconque $B$, que la gravité d'une partie quelconque est à la gravité du tout, comme la matière de la partie est à la matière totale, et que, par la troisième loi du mouvement, l'action et la réaction sont toujours égales; la planète $B$ gravitera à son tour vers toutes les parties de la planète $A$, et sa gravité vers une partie quelconque sera à sa gravité vers toute la planète, comme la matière de cette partie à la matière totale. - C.Q.F.D.

Cor. 1. La gravité vers toute une planète, est donc composée de la gravité vers toutes les parties. Nous en avons des exemples dans les attractions magnétiques et électriques. Car l'attraction vers le tout est composée des attractions vers chacune des parties. On verra qu'il en est de même dans la gravité, en supposant que plusieurs petites planètes s'unissent en un globe, et forment une grosse planète. Car on conçoit aisément par-là que la force totale doit naître de la force des parties composantes. Si quelqu'un objecte que selon cette loi tous les 
corps d'ici bas devraient graviter les uns vers les autres, et que cependant cette gravité mutuelle n'est pas sensible : je répondrai, que cette gravité mutuelle des corps étant à leur gravité vers la Terre, comme la masse de ces corps à la masse de la Terre, elle n'est pas à beaucoup près assez forte pour pouvoir être aperçue.

Cor. 2. La gravité vers chaque particule égale d'un corps, est réciproquement comme le carré des distances des lieux de ces particules. Ce qui est clair par le Cor. 3 de la Prop. 74 du premier Livre.

\section{Proposition VIII. — THÉORÈME VIII.}

Si la matière de deux globes qui gravitent l'un vers l'autre est homogène à égales distances de leurs centres : le poids de l'un de ces globes vers l'autre sera réciproquement comme le carré de la distance qui est entre leurs centres.

Après avoir trouvé que la gravité d'une planète entière est composée de celles de toutes ses parties, et que la force de chaque partie est réciproquement proportionnelle aux carrés des distances : j'ai voulu savoir si cette proportion réciproque doublée était suivie exactement pour la force totale composée de toutes les forces partiales, ou si elle ne l'était qu'à peu près. Car on pourrait croire que cette proportion, qui est assez exactement suivie à de grandes distances, devrait souffrir beaucoup d'altération près de la superficie des planètes, à cause de l'inégalité des distances des parties et de leurs différentes positions. Les Prop. 75 et 76 du premier Livre et leurs Corollaires m'ont fait voir que cette proportion était encore exactement observée dans le cas dont il s'agit.

Cor. 1. Par là on peut trouver les poids des corps sur diverses planètes et les comparer entre eux. Car les poids des corps égaux qui font leurs révolutions dans des cercles autour des planètes sont, par le Cor. 2 de la Prop. 4 du Liv. 1 comme les diamètres de ces cercles directement, et le carré des temps périodiques inversement; et leurs poids, à la surface de ces planètes, ou à quelques autres distances quelconques de leur centre, sont, par cette présente Proposition, plus grands ou moindres dans la raison doublée inverse des distances. Ainsi, le temps périodique de Vénus autour du Soleil étant de 224 jours et 16 heures $\frac{3}{4}$, celui du satellite le plus éloigné de Jupiter autour de cette planète 
de 16 jours et 16 heures $\frac{8}{15}$, le temps périodique du satellite d'Huygens autour de Saturne de 15 jours 22 heures $\frac{2}{3}$, et celui de la Lune autour de la Terre de 17 jours 7 heures 43 minutes, j'ai trouvé, en employant ces temps périodiques, et de plus la distance médiocre de Vénus au Soleil, la plus grande élongation héliocentrique du satellite de Jupiter le plus éloigné de cette planète au centre de Jupiter qui est $8^{\prime} 16^{\prime \prime}$, celle du satellite d'Huygens au centre de Saturne qui est de 3' 4" et celle de la Lune au centre de la Terre qui est de 10' 33", qu'à égale distance, les poids des corps égaux vers les centres du Soleil, de Jupiter, de Saturne et de la Terre, sont comme $1, \frac{1}{1067}, \frac{1}{3021}$ et $\frac{1}{169282}$ respectivement ; à des distances inégales ces poids varient en raison renversée du carré des distances : par exemple, les poids des corps égaux sur le Soleil, Jupiter, Saturne et la Terre aux distances 10 000, 997, 791 et 109 de leurs centres, c'est-à-dire, à leurs superficies, seront comme 10000 , 943,529 et 435 respectivement. On dira dans la suite ce que les corps pèsent à la surface de la Lune.

Cor. 2. On connaîtra aussi la quantité de matière que contient chaque planète. Car les quantités de matière dans les planètes sont comme leurs forces attractives à égales distances de leurs centres, c'est-à-dire, que les quantités de matière du Soleil, de Jupiter, de Saturne, et de la Terre sont comme $1, \frac{1}{1067}, \frac{1}{3021}$ et $\frac{1}{169282}$ respectivement. Si on trouve la parallaxe du Soleil plus grande ou plus petite que $10^{\prime \prime} 30^{\prime \prime}$, il faudra augmenter ou diminuer la quantité de matière de la Terre en raison triplée.

Cor. 3. On connaîtra aussi les densités des planètes. Car les poids des corps égaux et homogènes aux surfaces des sphères homogènes étant comme leurs diamètres, par la Prop. 71 du Liv. 1 les densités des sphères hétérogènes sont comme ces poids divisés par leurs diamètres. Or on a trouvé que les vrais diamètres du Soleil, de Jupiter, de Saturne, et de la Terre, sont l'un à l'autre comme $10000,997,791$ et 109, et que les poids sur ces planètes étaient comme $10000,943,529$ et 435 respectivement. Donc leurs densités sont comme 100, $94 \frac{1}{2}, 67$, et 400.

La densité de la Terre que ce calcul donne ne dépend point de la parallaxe du Soleil, mais elle est déterminée par la parallaxe de la Lune, ainsi elle l'est exactement. 
Le Soleil est donc un peu plus dense que Jupiter, Jupiter l'est plus que Saturne, et la Terre l'est quatre fois plus que le Soleil ; ce qu'il faut attribuer à la grande chaleur du Soleil, laquelle raréfie sa matière. La Lune est plus dense que la Terre comme on le verra dans la suite.

Cor. 4. Les planètes sont donc d'autant plus denses, qu'elles sont plus petites, toutes choses égales. Ainsi la force de la gravité à leur surface, approche plus de l'égalité. Les planètes qui sont plus près du Soleil sont aussi plus denses, toutes choses égales, ainsi Jupiter l'est plus que Saturne, et la Terre plus que Jupiter. Les planètes devaient donc être placées à différentes distances du Soleil, afin que chacune, à raison de sa densité, fut plus ou moins échauffée par le Soleil. Si la Terre était placée à l'orbe de Saturne, notre eau serait perpétuellement gelée, et si la Terre était dans l'orbe de Mercure, toute l'eau s'évaporerait dans l'instant. Car la lumière du Soleil, à laquelle la chaleur est proportionnelle, est sept fois plus dense dans Mercure que sur la Terre : et j'ai éprouvé par le Thermomètre que lorsque la chaleur était sept fois plus forte que celle du Soleil dans notre Été, elle faisait bouillir l'eau dans l'instant. Il n'est pas douteux que la matière de Mercure ne soit proportionnée à la chaleur qu'il éprouve, et que par conséquent elle ne soit plus dense que celle de la Terre; car plus la matière est dense, plus il faut de chaleur pour produire les mêmes effets.

\section{Proposition IX. - THÉORÈME IX.}

La gravité dans l'intérieur des planètes, décroît à peu près en raison des distances au centre.

Si la matière de la planète était d'une densité uniforme, cette Proposition serait vraie exactement, par la Prop. 73 du Liv. 1. Ainsi la loi de la pesanteur ne peut s'écarter de la proportion des distances que par l'inégalité de la densité.

\section{Proposition X. - ThÉORÈME X.}

Les mouvements des planètes peuvent se conserver très longtemps dans les espaces célestes. 
Dans le scholie de la Prop. 40 du Liv. 2 on a fait voir qu'un globe d'eau gelée mû librement dans notre air, perdrait par la résistance de l'air $\frac{1}{4586}$ partie de son mouvement en parcourant son demi-diamètre. La même proportion doit avoir lieu à peu près, dans des globes beaucoup plus grands, et qui se mouvraient avec beaucoup plus de vitesse que ceux dont on a parlé alors.

Mais le globe de la Terre est plus dense que s'il était entièrement formé d'eau, ce que je prouve ainsi. Si le globe de la Terre était d'eau, il y aurait des corps qui ayant moins de gravité spécifique surnageraient et reviendraient d'eux-mêmes à la superficie. Et par cette raison un globe composé de Terre qui serait entièrement entouré d'eau, surnagerait en quelque lieu s'il était plus léger que l'eau et cette eau s'amasserait vers le côté opposé. Il en est de même de notre Terre qui est en grande partie entourée par la mer. Si elle n'était pas plus dense que l'eau, elle surnagerait, et selon le degré de la légèreté spécifique elle sortirait en partie de l'eau qui se ramasserait toute dans les régions opposées.

Par le même raisonnement on doit conclure, que les taches du Soleil sont plus légères que la matière du Soleil sur laquelle elles nagent. Et dans la formation d'une planète quelconque qu'on suppose avoir été originairement fluide, la matière la plus pesante doit avoir été au centre. Ainsi comme la Terre est ordinairement à sa surface environ deux fois plus pesante que l'eau, et qu'en fouillant plus avant, elle est trois, quatre, et même cinq fois plus dense : il est vraisemblable qu'il y a environ cinq ou six fois plus de matière dans le globe de la Terre que s'il n'était formé que d'eau, surtout puisqu'on vient de faire, voir que la Terre est environ quatre fois plus dense que Jupiter. Si donc la matière de Jupiter est un peu plus dense que l'eau, il est clair que dans l'espace de trente jours, dans lesquels il parcourt la longueur de 459 de ses demi-diamètres, il ne perdrait que la dixième partie environ de son mouvement dans un milieu qui serait de la même densité que notre air. Or comme la résistance des milieux diminue avec leurs poids et leur densité, que l'eau, par exemple, qui est $13 \frac{3}{5}$ fois environ moins dense que le vif-argent, résiste $13 \frac{3}{5}$ fois moins que ce fluide ; et que l'air qui est 860 fois plus léger que l'eau résiste 860 fois moins : dans les cieux, où le poids du milieu dans lequel les planètes se meuvent diminue à l'infini, la résistance y doit être presque nulle. 
On a fait voir dans le Scholie de la Prop. 22. Liv. 2. que si on montait à la hauteur de deux cents milles au-dessus de la surface de la Terre, la densité de l'air à cette distance, serait à celle de l'air qui nous environne, comme 30 à 0,0000000000003998 , ou comme 75000000000000 à 1 environ. Ainsi la planète de Jupiter, en faisant sa révolution dans un milieu de cette densité, ne perdrait pas en 1000000 ans la $\frac{1}{1000000}$ partie de son mouvement par la résistance du milieu. Nous ne connaissons que l'air, les exhalaisons et les vapeurs, qui résistent près de la surface de la Terre puisque lorsqu'on les a ôté avec soin du récipient d'une machine pneumatique les corps y tombent librement, et sans éprouver aucune résistance sensible; de sorte que l'or même et une plume très légère étant jetés ensemble tombent avec une vitesse égale, et arrivent en même temps au fond de la machine en tombant de la hauteur de 4, 6 ou 8 pieds. Il est donc clair que les planètes pourront se mouvoir très longtemps sans éprouver de résistance sensible dans les espaces célestes vides d'air et d'exhalaisons.

\section{HYPOTHÈSE PREMIÈRE.}

Le centre du système du monde est en repos.

C'est ce dont on convient généralement, les uns seulement prétendent que la Terre est ce centre, et d'autres que c'est le Soleil. Voyons ce qui résulte de cette hypothèse.

\section{Proposition XL. - ThÉORÈME XI.}

Le centre commun de gravité du Soleil, de la Terre, et de toutes les planètes, est en repos.

Car ce centre, par le Cor. 4 des Lois, ou sera en repos, ou sera mû uniformément en ligne droite. Mais si ce centre avançait toujours, le centre du monde ne serait donc pas en repos, ce qui est contre l'hypothèse.

Proposition XII. - THÉORÈME XII.

Le Soleil est toujours en mouvement, mais il s'éloigne très peu du centre commun de gravité de toutes les planètes. 
Car puisque, par le Cor. 2 de la Prop. 8 la matière du Soleil est à la matière de Jupiter comme 1067 à 1, et que la distance de Jupiter au Soleil est au demi-diamètre du Soleil dans une raison un peu plus grande ; le commun centre de gravité du Soleil et de Jupiter tombera dans un point qui sera un peu au-dessus de la surface du Soleil. Par le même raisonnement, la matière du Soleil étant à la matière de Saturne comme 3021 à 1, et la distance de Saturne au Soleil étant au demidiamètre du Soleil dans une raison un peu moindre : le commun centre de gravité de Saturne et du Soleil tombera dans un point qui sera un peu au-dessus de la surface du Soleil. Et en suivant le même calcul on trouvera que si la Terre et toutes les planètes étaient placées d'un même côté du Soleil, le commun centre de gravité de tous ces astres s'éloignerait à peine du centre du Soleil d'un demi-diamètre de cet astre. Comme dans les autres cas la distance entre le centre du Soleil et le commun centre de gravité est encore moindre, et que ce commun centre de gravité est toujours en repos. Il arrive que le Soleil, selon la différente position des planètes, se meut successivement de tous les côtés, mais il ne s'écarte jamais que très peu du centre commun de gravité.

Cor. Le commun centre de gravité du Soleil, de la Terre, et de toutes les planètes, doit donc être regardé comme le centre du monde. Car la Terre, les planètes et le Soleil s'attirant mutuellement, ils sont toujours en mouvement par la force de leur gravité en vertu des lois du mouvement : ainsi leurs centres mobiles ne peuvent être pris pour le centre du monde, qui doit être en repos. Si le corps vers lequel la gravité entraîne plus fortement tous les autres devait être placé dans ce centre, (comme c'est l'opinion vulgaire) ce privilège appartiendrait au Soleil ; mais comme le Soleil se meut, il faut choisir pour le centre commun un point immobile duquel le centre du Soleil s'éloigne très peu, et duquel il s'éloignerait encore moins, si le Soleil était plus grand et plus dense, car alors il serait mû moins fortement.

\section{Proposition XIII. - ThÉORÈME XIII.}

Les planètes se meuvent dans des ellipses qui ont un de leurs foyers dans le centre du Soleil, et les aires décrites autour de ce centre sont proportionnelles au temps. 
Nous avons discuté ci-dessus ces mouvements d'après les Phénomènes. Les principes des mouvements une fois connus, donnent les mouvements célestes a priori. Ayant donc trouvé que les poids des planètes sur le Soleil sont réciproquement comme le carré de leurs distances à son centre, il est évident, par les Prop. 1 et 11, et par le Cor. 1 de la Prop. 13 du Livre 1, que si le Soleil était en repos, et que les planètes n'agissent point mutuellement les unes sur les autres, tous leurs orbes seraient des ellipses qui auraient le Soleil dans leur foyer commun, et elles décriraient autour de ce foyer des aires proportionnelles au temps. Or les actions mutuelles des planètes les unes sur les autres sont si faibles qu'elles peuvent être négligées, et, par la Prop. $66 \mathrm{du}$ Liv. 1 elles troublent moins la description de leurs ellipses autour du Soleil lorsqu'on suppose cet astre mobile, que si on le faisait immobile.

Cependant 1'action de Jupiter sur Saturne ne doit pas être absolument négligée : car la gravité vers Jupiter est à la gravité vers le Soleil (à distances égales) comme 1 à 1067 ; donc, dans la conjonction de Jupiter et de Saturne, la distance de Saturne à Jupiter étant à sa distance au Soleil à peu près comme 4 à 9 , la gravité de Saturne vers Jupiter sera à sa gravité vers le Soleil comme 81 à $16 \times 1067$ ou comme 1 à 211 à peu près. Et de là vient que l'orbe de Saturne est dérangé si sensiblement dans chaque conjonction avec Jupiter, que les Astronomes s'en aperçoivent. L'excentricité de cette planète est tantôt augmentée et tantôt diminuée selon sa situation dans ses conjonctions ; son aphélie avance quelquefois et quelquefois recule, et son mouvement moyen est tour à tour accéléré et retardé. Cependant tout le dérangement que l'attraction de Jupiter cause dans le mouvement de Saturne autour du Soleil, excepté dans le mouvement moyen, peut presque s'éviter en supposant le foyer inférieur de son orbite placé dans le centre commun de gravité de Jupiter et du Soleil (par la Prop. 67 du Liv. 1) alors lorsque ce dérangement est le plus grand, il passe à peine deux minutes. Et le plus grand dérangement dans le mouvement moyen surpasse à peine deux minutes par an.

Dans la conjonction de Jupiter et de Saturne les gravités accélératrices du Soleil vers Saturne, de Jupiter vers Sarurne et de Jupiter vers le Soleil sont à peu près comme 16,81 et $\frac{16 \times 81 \times 3021}{25}$ ou 156609 : 
ainsi la différence des gravités du Soleil et de Jupiter vers Saturne est à la gravité de Jupiter vers le Soleil comme 65 à 156 609, ou comme 1 à 2409. La plus grande force de Saturne pour troubler les mouvements de Jupiter est proportionnelle à cette différence, aussi le dérangement de l'orbe de Jupiter est-il beaucoup moindre que celui de l'orbe de Saturne.

Les dérangements qu'éprouvent les orbes des autres planètes par leurs actions mutuelles sont beaucoup moins considérables si on en excepte l'orbe de la Terre que la Lune dérange sensiblement. Le commun centre de gravité de la Terre et de la Lune décrit autour du Soleil une ellipse dont cet astre est le foyer, et donc les aires décrites par ce centre sont proportionnelles au temps : la Terre fait sa révolution autour de ce centre commun dans un mois.

\section{Proposition XIV. - THÉORÈME XIV.}

L'aphélie et les nœuds des orbites sont en repos.

Les aphélies sont en repos par la Prop. 11 du Liv. 1 et par la première du même livre les plans des orbes sont aussi immobiles, et par conséquent les nœuds. Il faut avouer cependant que les actions des planètes et des comètes les unes sur les autres, peuvent causer quelques inégalités tant dans les aphélies que dans les nœuds, mais ce sont des inégalités assez petites pour qu'il soit permis de les négliger.

Cor. 1. Les étoiles fixes sont aussi en repos, car elles conservent les mêmes positions par rapport aux nœuds et aux aphélies.

Cor. 2. Donc puisque le mouvement annuel de la Terre ne leur cause point de parallaxe sensible, leurs forces attractives ne produisent point d'effets sensibles dans la région de notre système à cause de la distance immense de ces corps. Peut-être les étoiles fixes, qui sont également dispersées dans toutes les parties du ciel, détruisent-elles leurs forces mutuelles par leurs attractions contraires, selon la Prop. $70 \mathrm{du}$ Liv. 1. 


\section{SCHOLIE.}

Comme 1'action mutuelle des planètes qui sont le plus près du Soleil, telles que Vénus, Mercure, la Terre et Mars sont presque insensibles à cause de la petitesse de ces planètes : leurs nœuds et leurs aphélies sont en repos, à l'altération près que peut y apporter l'action de Saturne, de Jupiter et des autres corps placés au-dessus d'elles. En ayant égard à cette altération, on trouve, par la théorie de la gravité, que leurs aphélies se meuvent un peu en conséquence par rapport aux fixes, et cela dans la proportion sesquiplée des distances de ces planètes au Soleil. De sorte que si l'aphélie de Mars fait 33' 20" en cent ans, en conséquence par rapport aux fixes : les aphélies de la Terre, de Vénus, et de Mercure seront dans le même espace de cent ans $17^{\prime} 40^{\prime \prime}$, $10^{\prime} 53^{\prime \prime}$ et $4^{\prime} 16^{\prime \prime}$ respectivement. Mais on ne fait pas attention dans cette Proposition à ces mouvements qui sont presque insensibles.

\section{Proposition XV. - Problème I.}

Trouver les diamètres principaux des orbes.

Il faut les prendre en raison sesquiplée des temps périodiques, par la Prop. 15 du Liv. 1. Ensuite, par la Prop. 60 du Liv. 1. il faut augmenter le diamètre de chacun des orbes dans la raison qu'il y a entre la masse de la planète ajoutée à celle du Soleil, et la première des deux moyennes proportionnelles entre cette somme et le Soleil.

Proposition XVI. - Problème II.

Trouver les excentricités et les aphélies des orbes.

Ce Problème se résout par la Prop. 18 du Liv. 1.

\section{Proposition XVII. - THÉORÈME XV.}

Les mouvements diurnes des planètes sont uniformes, et la libration de la Lune vient de son mouvement diurne.

Cela est clair par la première loi du mouvement et par le Cor. 22 de la Prop. 66 Liv. 1. 
Jupiter par rapport aux fixes fait sa révolution diurne en $9^{\mathrm{h}} 56^{\prime}$, Mars en $24^{\mathrm{h}} 39^{\prime}$, Vénus en $23^{\mathrm{h}}$ environ, la Terre en $23^{\mathrm{h}} 56^{\prime}$, le Soleil en 25 jours $\frac{1}{2}$ et la Lune en 27 jours $7^{\mathrm{h}} 43^{\prime}$, c'est ce que les Phénomènes prouvent. Les taches du Soleil revenant sur son disque dans la même situation au bout de $27 \mathrm{j} . \frac{1}{2}$ par rapport à la Terre ; il faut que le Soleil fasse sa révolution par rapport aux fixes en $25 \mathrm{j}$. $\frac{1}{2}$ environ. Et comme le jour de la Lune par la révolution uniforme autour de son axe est d'un mois, sa même face doit regarder toujours la Terre à la différence près qui est produite par l'excentricité de son orbite. C'est là la libration de la Lune en longitude : quant à sa libration en latitude, elle dépend de la latitude de la Lune, et de l'inclinaison de son axe au plan de l'écliptique.

Mercator a amplement expliqué la théorie de cette libration de la Lune d'après mes lettres dans son Astronomie publiée au commencement de l'année 1676.

Le satellite le plus éloigné de Saturne paraît tourner autour de son axe d'un mouvement semblable, et présenter toujours le même côté à Saturne; car toutes les fois qu'il approche de la partie orientale de l'orbe de cette planète, on le voit à peine, et souvent il disparaît entièrement : ce qui peut venir de ce qu'il présente alors à la Terre une partie de son disque dans laquelle il se trouve des taches, comme Cassini l'a remarqué.

Le satellite le plus éloigné de Jupiter paraît tourner aussi de même autour de son axe, car il a, dans la partie de son disque opposée à Jupiter, une tache que l'on voit comme si elle était dans le disque même de Jupiter, toutes les fois que ce satellite passe entre Jupiter et nos yeux.

\section{Proposition XVIII. — ThÉorÈME XVI.}

Les axes des planètes sont plus petits que les rayons de leurs équateurs.

Si les planètes n'avaient point le mouvement journalier de rotation autour de leur axe, elles devraient être sphériques à cause de l'égale gravité de leurs parties. Le mouvement de rotation fait que les parties qui s'éloignent de l'axe font effort pour monter vers l'équateur. Et par 
conséquent, si la matière dont elles sont composées était fluide, son élévation vers l'équateur augmenterait le diamètre de ce cercle, et son abaissement vers les Pôles diminuerait l'axe. Aussi les observations astronomiques nous apprennent-elles que dans Jupiter le diamètre qui va d'un pôle à l'autre est plus court que celui qui va de l'Orient à l'Occident. Par le même raisonnement, on verra que si notre Terre n'était pas un peu plus haute à l'équateur qu'aux pôles, les mers s'affaissant vers les pôles, et s'élevant vers l'équateur inonderaient toutes ces régions.

\section{Proposition XIX. - ProblèMe III.}

Trouver la proportion des axes d'une planète.

Norvood, notre compatriote, vers l'année 1635 trouva en mesurant un espace de 905751 pieds anglais entre Londres et Yorck, et en observant la différence des latitudes de ces deux villes qui est de $2^{\circ} 28^{\prime}$, que le degré avait 367196 pieds anglais, c'est-à-dire 57300 toises de Paris.

Picart en mesurant un arc de $1^{\circ} 22^{\prime} 55^{\prime \prime}$ dans le méridien entre Amiens et Malvoisine, trouva que le degré avait 57060 toises de Paris, Cassini le père mesura dans le méridien la distance entre la ville de Collioure en Roussillon et l'observatoire de Paris : et son fils ajouta à cette mesure celle de la distance entre l'observatoire de Paris, et la tour de Dunkerque : la distance totale était de $486156 \frac{1}{2}$ toises, et la différence des latitudes des villes de Collioure et de Dunkerque de $8^{\circ}$ 31' $11 \frac{1}{2}$ ", ce qui donne l'arc d'un degré de 57061 toises de Paris. De ces mesures on conclut la circonférence de la Terre de 123249600 pieds de Paris, et son demi-diamètre de 19615800 pieds, en supposant que la Terre soit sphérique.

On a vu ci-dessus que dans la latitude de Paris les corps graves en tombant parcourent 15 pieds 1 pouces et $1 \frac{7}{9}$ lignes ou $2173 \frac{7}{9}$ lignes en une seconde. Mais le poids des corps diminue par le poids de l'air qui les environne; supposons que cette diminution soit la $\frac{1}{11000}^{\mathrm{e}}$ partie du poids total, le corps en tombant dans le vide parcourrait 2174 lignes en une seconde. 
Un corps qui circulerait dans un cercle à la distance de 19615800 pieds du centre, et qui serait la révolution uniformément en $23^{\text {h }} 56^{\prime} 4^{\prime \prime}$ sidérales, décrirait un arc de 1433,46 pieds en une seconde, le sinus verse de cet arc est de 0,0523656 pieds ou de 7,54064 lignes. Ainsi la force avec laquelle les graves descendent à la latitude de Paris, est à la force centrifuge des corps sous l'équateur causée par le mouvement de rotation de la Terre, comme 2174 à 7,54064.

La force centrifuge des corps sous l'équateur, est à la force centrifuge par laquelle les corps tendent à s'éloigner perpendiculairement de la Terre à la latitude de Paris qui est de $48^{\circ} 50^{\prime} 10^{\prime \prime}$ en raison doublée du rayon au sinus du complément de cette latitude, c'est-à-dire, comme 7,54054 à 3,267. En ajoutant cette force à la force qui fait descendre les graves à la latitude de Paris, la chute des graves produite à cette latitude par la force totale de la gravité sera dans une seconde de 2177,167 lignes ou 15 pieds 1 pouce, 5,267 lignes de Paris. Et la force totale de la gravité dans cette latitude sera à la force centrifuge des corps sous l'équateur comme 2177,267 à 7,54064 ou comme 289 à 1.

(Fig. 1)

Si présentement $A P B Q$ représente la Terre non supposée sphérique comme auparavant, mais formée par la révolution d'une ellipse autour de son petit axe $P Q$, et que ACQqca, soit un canal plein d'eau depuis le pôle $Q q$ jusqu'au centre $C c$, et depuis ce centre jusqu'à l'équateur $A a$ : le poids de l'eau dans la branche ACca du canal, doit être au poids de l'eau dans l'autre branche QCcq comme 289 à 288 à cause que la force centrifuge qui vient du mouvement circulaire soutient et ôte du poids de l'eau une partie sur 289 et que par conséquent les 288 parties d'eau qui sont dans la branche ACca soutiennent les 289 de l'autre.

En suivant la méthode du Cor. 2 de la Prop. 91 du Livre 1, je trouve que si la Terre était composée d'une matière homogène, qu'elle fut privée de tout mouvement, et que son axe $P Q$ fut à son diamètre $A B$ comme 100 à 101 : la gravité au lieu $Q$ de la Terre serait à la gravité dans le même lieu $Q$ d'une sphère décrite du centre $C$ et du rayon $P C$ ou $Q C$, comme 126 à 125 .

Par le même raisonnement, on trouvera que la gravité dans le lieu $A$ d'un sphéroïde décrit par la révolution de l'ellipse $A P B Q$ autour de 
son axe $A B$, est à la gravité au même lieu $A$ dans une sphère décrite du centre $C$ et du rayon $A C$, comme 125 à 126. De plus la gravité au lieu $A$ de la Terre est moyenne proportionnelle entre les gravités dans ce sphéroïde et dans cette sphère : à cause que la sphère, en diminuant le diamètre $P Q$ dans la raison de 101 à 100 , se changerait dans la figure de la Terre; et que cette figure en diminuant dans la même raison le diamètre perpendiculaire aux deux diamètres $A B, P Q$, se changerait dans le sphéroïde décrit par la révolution de l'ellipse $A B P Q$ autour de $A B$; et dans l'un et l'autre cas, la gravité en A diminuerait dans la même raison à peu près.

Enfin la gravité en $A$ dans la sphère dont le centre est $C$ et le rayon $A C$, est à la gravité au même lieu $A$ sur la Terre, comme 126 à $125 \frac{1}{2}$, et la gravité au lieu $Q$ dans la sphère dont le centre est $C$ et le rayon $Q C$ est à la gravité au lieu $A$ dans la sphère dont le centre est $C$ et le rayon $A C$, en raison des diamètres, (par la Prop. 72 du Liv. 1) c'est-àdire, comme 100 à 101. Joignant donc ces trois raisons 126 à 125, 126 à $125 \frac{1}{2}$ et 100 à 101 , la gravité sur la Terre au lieu $Q$ sera à la gravité sur la Terre au lieu $A$, comme $126 \times 126 \times 100$ à $125 \times 125 \frac{1}{2} \times 101$, ou comme 501 à 500 .

Or, comme (par le Cor. 3 de la Prop. 91 du Liv. 1) la gravité dans l'un ou l'autre branche ACca ou QCcq du canal est comme la distance des lieux au centre de la Terre; si ces branches sont séparées en parties proportionnelles aux touts par des surfaces transversales et équidistantes, les poids d'un nombre quelconque de parties de l'une de ces branches, seront aux poids d'autant de parties dans l'autre branche en raison composée des quantités de matière et des forces accélératrices, c'est-à-dire, de la raison de 101 à 100 et de celle de 500 à 501, ou, ce qui revient au même, en raison simple de 505 à 501 . Donc, si la force centrifuge d'une partie quelconque de la branche ACca, laquelle vient du mouvement diurne, était au poids de la même partie, comme 4 à 505 , en sorte que du poids de cette partie divisée en 505, sa force centrifuge en ôtât 4 ; les poids seraient égaux dans l'une et l'autre branche, et par conséquent le fluide resterait en équilibre.

Mais la force centrifuge d'une partie quelconque est au poids de cette même partie comme 1 à 289 , c'est-à-dire, que la force centrifuge qui devrait être la $\frac{4}{505}^{\mathrm{e}}$ partie du poids n'en est que la $\frac{1}{289}^{\mathrm{e}}$ partie, ainsi 
on peut dire, par une simple analogie, si la force centrifuge $\frac{4}{505}$ fait que la hauteur de l'eau dans la branche ACca surpasse la hauteur de l'eau dans la branche QCcq d'une centième partie de toute la hauteur : la force centrifuge $\frac{1}{289}$ fera que l'excès de la hauteur dans la branche ACca ne sera que $\frac{1}{229}^{\mathrm{e}}$ partie de la hauteur de l'eau dans l'autre branche QCcq. Et le diamètre de la Terre qui passe par ses pôles sera au diamètre de l'équateur comme 229 à 230. Ainsi, comme le demidiamètre médiocre de la Terre est, selon la mesure de Picart, de 19615800 pieds de Paris ou de 3923,16 milles, (suppose que le mille soit de 5000 pieds) la Terre sera plus haute à l'équateur qu'aux pôles de 85472 pieds, ou de $17 \frac{1}{10}$ milles, et sa hauteur à l'équateur sera de 19658600 pieds environ, et de 19573000 aux pôles.

Si la planète est plus petite ou plus grande que la Terre, mais que sa densité, et le temps périodique de sa révolution diurne soient les mêmes, la proportion de la force centrifuge à la gravité demeurera la même, et par conséquent la proportion entre l'axe et le diamètre de l'équateur sera aussi la même.

Mais si le mouvement diurne est accéléré ou retardé dans une raison quelconque, il augmentera ou diminuera la force centrifuge dans la raison doublée de cette raison, et par conséquent la différence des diamètres augmentera ou diminuera dans cette même raison doublée à peu près. Si la densité de la planète augmente ou diminue dans une raison quelconque, la gravité vers cette planète augmentera ou diminuera dans la même raison. Mais la différence des diamètres diminuera au contraire en raison de l'augmentation de la gravité ou augmentera en raison de la diminution de la gravité. Ainsi comme la Terre fait sa révolution en $23^{\mathrm{h}} 56^{\prime}$ et Jupiter en $9^{\mathrm{h}} 56^{\prime}$, par rapport aux fixes, et que par conséquent les carrés des temps sont comme 29 à 5 , et les densités comme 400 à $94 \frac{1}{2}$ : la différence des diamètres de Jupiter sera à son petit diamètre comme $\frac{29}{5} \times \frac{400}{94 \frac{1}{2}} \times \frac{1}{229}$ à 1 , ou comme 1 à $9 \frac{1}{3}$; à peu près. Le diamètre de Jupiter de l'Orient à l'Occident est donc à son diamètre entre les pôles comme $10 \frac{1}{3}$ à $9 \frac{1}{3}$ à peu près. Donc, puisque son plus grand diamètre est de 37", son petit diamètre entre ses pôles sera de $33^{\prime \prime} 25^{\prime \prime \prime}$ et ajoutant 3 " à peu près pour la lumière erratique, les diamètres apparents de cette planète seront de $40^{\prime \prime}$ et $36^{\prime \prime}$ 
$25^{\prime \prime \prime}$ à peu près : c'est-à-dire, qu'ils seront l'un à l'autre comme $11 \frac{1}{6}$ à $10 \frac{1}{6}$ à peu près. Mais ce rapport ne doit avoir lieu qu'en supposant toute la matière de Jupiter d'une égale densité ; car si elle était plus dense vers le plan de l'équateur que vers les pôles, ses diamètres pourraient être l'un à l'autre comme 12 à 11 , ou comme 13 à 12 , ou même comme 14 à 13. Cassini a observé dans l'année 1691 que le diamètre de Jupiter de l'Orient à l'Occident surpassait son autre diamètre environ d'une de ses quinzièmes parties. Notre compatriote Pound avec un télescope de 123 pieds et un excellent Micromètre, ayant mesuré les diamètres de Jupiter en 1719, les trouva tels qu'ils sont marqués dans la table suivante.

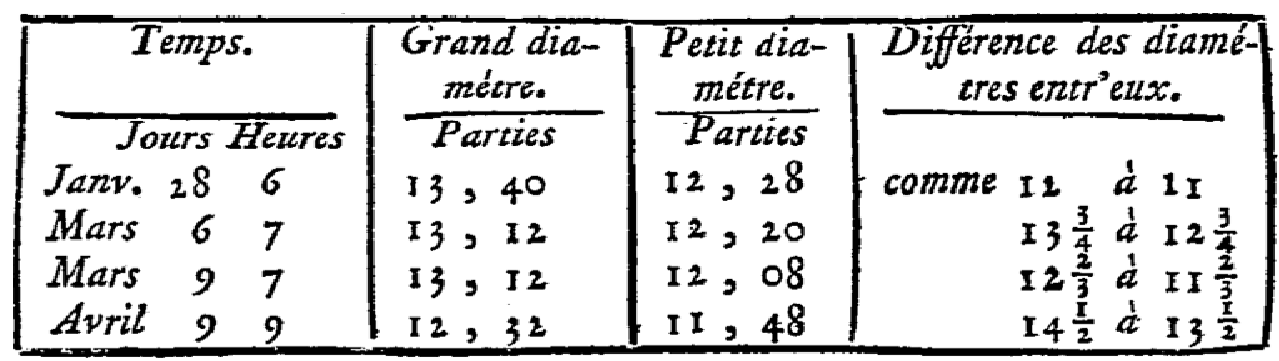

Cette théorie s'accorde avec les Phénomènes; car l'équateur des planètes étant beaucoup plus exposé que les autres parties à l'action du Soleil, la matière qui y est, pour ainsi dire, plus cuite doit y être plus dense que vers les pôles.

Que la gravité diminue sous l'équateur par la rotation diurne de notre Terre, et que par conséquent elle doive être plus élevée vers l'équateur qu'aux pôles, (si sa matière est d'une densité uniforme) c'est ce qui paraîtra clairement par les expériences des pendules que je vais rapporter dans la Proposition suivante.

\section{Proposition XX. - ProblèME IV.}

Trouver et comparer entre eux les poids des corps dans les diverses régions de la Terre.

Comme les poids de l'eau renfermée dans les branches inégales du canal ACQqca sont égaux; et que les poids de ses parties, qui sont proportionnelles aux branches, et situées de même dans leur totalité, sont entre eux comme les poids entiers, et que par conséquent ils sont égaux entre eux; les poids des parties égales et également situées dans 
ces branches, seront réciproquement comme ces branches, c'est-àdire, comme 230 à 229. Il en est de même de tous les corps quelconques homogènes égaux, et qui seront situées semblablement dans les branches de ce canal; leurs poids seront réciproquement comme ces branches, c'est-à-dire, réciproquement comme les distances de ces corps au centre de la Terre. C'est pourquoi, les poids des corps situés dans les parties supérieures de ces canaux, ou à la surface de la Terre, seront entre eux réciproquement comme leur distance à son centre. Par le même raisonnement, les poids, dans quelque région de la Terre que ce soit, sont réciproquement comme les distances des lieux au centre de la Terre; et par conséquent, en supposant que la Terre soit un sphéroïde, leur proportion est donnée.

On tire de là ce théorème, que l'augmentation du poids, en allant de l'équateur vers les pôles, doit être à peu près comme le sinus verse du double de la latitude, ou, ce qui est la même chose, comme le carré du sinus droit de la latitude. Les arcs des degrés de latitude augmentent à peu près dans la même raison dans le méridien. Ainsi la latitude de Paris étant de $48^{\circ} 50^{\prime}$, celle des lieux situés sous l'équateur de $00^{\circ}$ $00^{\prime}$, et celle des lieux situés aux pôles de $90^{\circ}$, les sinus verses des arcs doubles étant par conséquent de 11334 , 00000, et 20000 , pour le rayon de 10000 ; et la gravité aux pôles étant étant à la gravité sous l'équateur comme 230, à 229, ou, ce qui revient au même, l'excès de la gravité aux pôles étant à la gravité sous l'équateur comme 1 à 229 : on trouvera que l'excès de la gravité dans la latitude de Paris, est à la gravité sous 1 équateur, comme $1 \times \frac{11334}{20000}$ à 229 , ou comme 5667 à 2290000 . Donc les gravités totales dans ces lieux, seront l'une à l'autre comme 2295667 à 2290000 . Or comme les longueurs des pendules qui font leurs oscillations en temps égaux, sont en raison directe des gravités, et qu'à la latitude de Paris la longueur du pendule qui bat les secondes est de 3 pieds de Paris $8 \frac{1}{2}$ lignes, ou plutôt de 3 pieds $8 \frac{5}{9}$ lignes, à cause du poids de l'air : la longueur du pendule sous l'équateur sera moindre que la longueur du pendule synchrone à la latitude de Paris. Et cette différence sera d'une ligne et 87 millièmes de lignes. C'est par un semblable calcul qu'on a dressé la table suivante. 


\begin{tabular}{|c|c|c|}
\hline $\begin{array}{c}\text { Latitude } \\
\text { du lieu. }\end{array}$ & $\begin{array}{c}\text { Longueur du Pen } \\
\text { dule. }\end{array}$ & $\begin{array}{c}\text { Mefure d'un dégr } \\
\text { du Méridien. }\end{array}$ \\
\hline$\overline{\text { Dégrés. }}$ & Fteds. Lignis. & Toijes. \\
\hline 0 & 37,468 & 56637 \\
\hline ro & $\begin{array}{lll}3 & 7,482 \\
3 & 7,526\end{array}$ & $\begin{array}{l}56642 \\
56659\end{array}$ \\
\hline 15 & 7,596 & 56687 \\
\hline 20 & 7,692 & 56724 \\
\hline 25 & 7,812 & $5^{6} 7^{69}$ \\
\hline 35 & $\begin{array}{lll}3 & 7,948 \\
3 & 8,099\end{array}$ & $\begin{array}{l}50023 \\
56882\end{array}$ \\
\hline 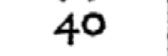 & $8,26 I$ & 56945 \\
\hline$\pi$ & 8,294 & 56958 \\
\hline 2 & 8,327 & 56971 \\
\hline 4 & $\begin{array}{l}8,361 \\
8, \quad 394\end{array}$ & 56997 \\
\hline & 8,428 & $570: 0$ \\
\hline & 461 & 57022 \\
\hline 7 & 8,494 & 57035 \\
\hline 9 & 8,561 & 57061 \\
\hline 50 & 8,594 & 57074 \\
\hline 55 & $\begin{array}{l}8,756 \\
8,007\end{array}$ & $\begin{array}{l}57137 \\
57196\end{array}$ \\
\hline 65 & 9,044 & 57250 \\
\hline 70 & 9,162 & 57295 \\
\hline 75 & 9,258 & $\begin{array}{l}57332 \\
57360\end{array}$ \\
\hline 8 & 9,372 & 57377 \\
\hline & 39,307 & \\
\hline
\end{tabular}

On voit par cette table que l'inégalité des degrés est si petite, que dans la géographie on peut supposer la Terre sphérique : surtout si la matière est plus dense vers l'équateur que vers les pôles.

Quelques Astronomes envoyés dans des régions fort éloignées pour faire des observations astronomiques, observèrent que le mouvement des horloges à pendule était plus lent vers l'équateur que dans nos pays. M. Richer fut le premier qui fit cette observation dans l'île de Cayenne en 1672. En observant au mois d'août le passage des fixes par le méridien, il trouva que sa pendule retardait sur le moyen mouvement du Soleil, et que la différence par jour était de 2' 28". Ensuite ayant fait osciller un pendule simple de sorte que ses vibrations fussent isochrones à celles de la pendule qui était excellente, il détermina la longueur du pendule simple, et il répéta les expériences plusieurs 
fois chaque semaine pendant 10 mois. Étant ensuite retourné en France il compara la longueur de ce pendule avec celle du pendule qui bat les secondes à Paris (lequel avait 3 pieds de Paris et 8 lignes $\frac{3}{5}$ ) et il trouva que le pendule sous l'équateur était plus court qu'à Paris d'une ligne et un quart de ligne.

Depuis ce temps, Halley notre compatriote trouva vers l'année 1677 qu'à l'île de Sainte Hélène le mouvement de sa pendule était plus lent qu'à Londres, il n'en détermina pas la différence, mais il raccourcit son pendule de plus de la huitième partie d'un pouce, c'est-àdire, d'une ligne et demie. Pour faire cette opération, comme la longueur de la vis vers le bas du pendule n'était pas suffisante, il mit un anneau de bois à la boîte de la vis, et il y suspendit le poids du pendule.

Ensuite dans l'année 1682 MM. Varin et Deshayes déterminèrent la longueur du pendule qui bat les secondes à l'Observatoire de Paris, de 3 pieds de Paris 8 lignes et $\frac{1}{9}$, et dans l'île de Gorée ils trouvèrent par la même méthode que la longueur du pendule synchrone était de 3 pieds 6 lignes et $\frac{1}{9}$, ainsi la différence était de deux lignes. La même année, aux îles de la Guadeloupe et de la Martinique, ils trouvèrent la longueur du pendule synchrone de 3 pieds 6 lignes $\frac{1}{2}$.

M. Couplet le fils en 1697 au mois de Juillet, régla sa pendule sur le moyen mouvement du Soleil à l'observatoire de Paris, de sorte que pendant un temps assez long, elle s'accordait parfaitement avec le mouvement du Soleil, et étant à Lisbonne au mois de Novembre suivant il trouva que cette même pendule retardait, et que la différence était de $2^{\prime} 13^{\prime \prime}$ en 24 heures. Au mois de mars suivant, il trouva, qu'à Paraïbe son horloge retardait sur Paris de $4^{\prime} 12^{\prime \prime}$ en 24 heures. Et il assure que le pendule qui battait les secondes à Lisbonne était plus court que celui qui les battait à Paris de 2 lignes $\frac{1}{2}$ et que celui qui les battait à Paraïbe était plus court que celui qui les battait à Paris de 3 lignes $\frac{2}{3}$. Il aurait déterminé plus exactement ces différences s'il eût fait celle de Lisbonne de 1 ligne $\frac{1}{3}$ et celle de Parä̈be de 2 lignes $\frac{5}{9}$, car ces différences répondent respectivement à $2^{\prime} 13^{\prime \prime}$ et à $4^{\prime} 12^{\prime \prime}$ qui sont les différences qu'il avait remarquées entre les temps marqués par son horloge, ainsi on ne doit pas beaucoup ajouter de foi à ces observations grossières. 
Les années suivantes, c'est-à-dire, en 1699 et en 1700 M. Deshayes étant de nouveau en Amérique, détermina la longueur du pendule qui bat les secondes dans les îles de Cayenne et de Grenade un peu moindre de 3 pieds 6 lignes $\frac{1}{2}$. Dans l'île de S. Christophe, il trouva cette longueur de 3 pieds 6 lignes $\frac{3}{4}$. Et dans l'île de S. Domingue de 3 pieds 7 lignes.

En l'année 1704 le P. Feuillée trouva à Portobello en Amérique, la longueur du pendule qui bat les secondes de 3 pieds de Paris, 5 lignes et $\frac{7}{12}$, c'est-à-dire, près de 3 lignes moindre qu'à Paris, mais il dut y avoir de l'erreur dans son observation, car étant allé ensuite à la Martinique, il trouva que la longueur du pendule isochrone n'était que trois pieds de Paris 5 lignes et $\frac{10}{12}$.

Or la latitude méridionale de Paraïbe est de $6^{\circ} 38^{\prime}$, la latitude septentrionale de Portobello de $9^{\circ} 33^{\prime}$, et les latitudes septentrionales des îles de Cayenne, de Gorée, de la Guadeloupe, de la Martinique, de Grenade, de $S$. Christophe, et de $S$. Domingue, sont respectivement de $4^{\circ} 55^{\prime}, 14^{\circ} 40^{\prime}, 14^{\circ} 00^{\prime}, 14^{\circ} 44^{\prime}, 12^{\circ} 6^{\prime}, 17^{\circ} 19^{\prime}$, et de $19^{\circ} 48^{\prime}$; et les excès de la longueur du pendule de Paris sur les longueurs des pendules isochrones observées dans ces latitudes, sont un peu plus grands que ne les donne la table des longueurs du pendule calculée ci-dessus. Ainsi la Terre doit être un peu plus élevée à l'équateur que ce calcul ne l'a donné, et sa matière doit être plus dense à son centre que près de la superficie, supposé cependant que la chaleur de la Zone torride n'ait pas un peu augmenté la longueur du pendule.

M. Picart a observé qu'une barre de fer, qui pendant la gelée était longue d'un pied, devenait, étant échauffée par le feu, d'un pied et un quart de ligne. Et M. de la Hire a remarqué depuis, qu'une barre de fer qui avait six pieds pendant l'hiver, devenait de six pieds et $\frac{2}{3}$ de ligne lorsqu'elle était exposée au Soleil de l'Été.

Dans le premier cas, la chaleur fut plus grande que dans le second, et dans celui-ci la chaleur fut plus grande que celle des parties externes du corps humain, car les métaux acquièrent une grande chaleur lorsqu'ils sont exposés au Soleil de l'Été. Mais le pendule d'une horloge n'est jamais exposé au Soleil de l'Été, et n'atteint même jamais la chaleur des parties externes du corps humain. Ainsi le pendule de l'horloge dont la longueur était de trois pieds, n'a jamais pu devenir 
plus long l'Été que l'Hiver, que d'un quart de ligne, et par conséquent on ne peut attribuer les différences qui se trouvent entre les longueurs des pendules isochrones en différentes régions à la différente chaleur des climats. Elle ne peut être attribuée non plus aux erreurs glissées dans les observations des Astronomes français, car quoiqu'elles ne s'accordent pas parfaitement entre elles, cependant les différences sont si petites qu'on peut les négliger. Ces observations s'accordent toutes à donner les pendules isochrones plus courts vers l'équateur qu'à l'observatoire de Paris, et selon toutes ces observations, cette différence n'est pas moindre que d'une ligne et un quart, et elle ne passe pas 2 lignes $\frac{2}{3}$.

Dans les observations de M. Richer à Cayenne, la différence fut d'une ligne et un quart, dans celle de M. Deshayes la différence corrigée fut d'une ligne et demie, ou d'une ligne trois quarts, dans les autres observations qui sont moins exactes elle était environ de deux lignes; et ces différences doivent être attribuées, partie aux erreurs commises dans les observations, partie à la dissemblance des parties internes de la Terre, et à la différente hauteur des montagnes, et partie enfin à la différente température de l'air.

Une barre de fer longue de trois pieds est plus courte en Angleterre l'Hiver que l'Été de la sixième partie d'une ligne, autant que j'en puis juger ; ainsi ôtant cette différence causée par la chaleur, d'une ligne et un quart, qui est la différence trouvée par M. Richer, il restera toujours une différence de $1 \frac{1}{12}$ ligne, qui approche assez de $1 \frac{87}{1000}$ trouvée ci-dessus par la théorie. Richer répéta ses observations à la Cayenne toutes les semaines pendant 10 mois, et il compara les longueurs du pendule à Cayenne avec les longueurs du même pendule en France déterminées de même. Les autres observateurs n'avaient point fait leurs observations avec tant de soin et de précaution, si donc on regarde les observations de M. Richer comme exactes, il s'ensuivra que la Terre doit être plus haute à l'équateur qu'aux pôles de 17 milles environ, comme la théorie précédente l'a donné.

\section{Proposition XXI. - THÉORÈME XVII.}

Les points équinoxiaux rétrogradent, et l'axe de la Terre, à chaque révolution annuelle, a une nutation par laquelle il 
s'incline deux fois vers l'écliptique et retourne deux fois à sa première position.

C'est ce qui est prouvé par le Cor. 20 de la Prop. 66 du Liv. 1 mais ce mouvement de nutation doit être très faible, et on peut à peine s'en apercevoir.

\section{Proposition XXII. — THÉORÈME XVIII.}

Tous les mouvements de la Lune, et toutes ses inégalités sont une suite et se tirent des principes qu'on a posés cidessus.

Pendant que les grandes planètes sont portées autour du Soleil, elles peuvent emporter dans leur révolution d'autres planètes plus petites, qui tournent autour d'elles dans des ellipses dont le foyer est placé dans le centre des grandes planètes, ce qui est clair par la Prop. $65 \mathrm{du}$ Liv. 1. Les mouvements de ces petites planètes doivent être troublés de plusieurs façons par l'action du Soleil qui doit causer des inégalités dans leur mouvement telles qu'on en remarque dans notre Lune ; car dans les syzygies cette planète (selon les Cor. 2, 3, 4 et 5 de la Prop. 66) se meut plus vite et décrit autour de la Terre des aires plus grandes en temps égaux que dans les quadratures, et alors elle parcourt un orbe moins courbe, et approche par conséquent plus près de la Terre, à moins que son mouvement excentrique ne fasse un effet contraire. Car l'excentricité de la Lune est la plus grande (par le Cor. 9 de la Prop. 66) lorsque son apogée est dans les syzygies, et elle est la moindre lorsque l'apogée est dans les quadratures; de sorte que la Lune va plus vite et est plus près de la Terre dans son périgée ; et elle va plus lentement, et est plus loin de nous dans son apogée, lorsqu'elle est dans les syzygies que lorsqu'elle est dans les quadratures. De plus, l'apogée avance, et les nœuds rétrogradent, mais d'un mouvement inégal ; l'apogée (par les Cor. 7 et 8, de la Prop. 66) avance plus vite dans les syzygies, et rétrograde plus lentement dans ses quadratures, et l'excès du mouvement progressif sur la rétrogradation se fait, pour l'année entière, en conséquence. Mais les nœuds (par le Cor. 2 de la Prop. 66) sont en repos dans leurs syzygies, et rétrogradent très vite dans leurs quadratures. Quant à la plus grande latitude de la Lune, elle est plus grande dans ses quadratures (par le Cor. 10 de la Prop. 66) que dans ses syzygies : et le moyen mouvement est plus lent dans le 
périhélie de la Terre (par le Cor. 6 de la Prop. 66) que dans son aphélie. Ce sont là les inégalités les plus remarquables que les Astronomes aient observées dans le mouvement de la Lune.

Il y en a encore quelques-unes qui n'avaient pas été observées par les premiers Astronomes, et qui troublent tellement les mouvements lunaires, que jusqu'à présent, on n'avait pu les réduire à aucune règle certaine. Telles sont les vitesses ou les mouvements horaires de l'apogée et des nœuds de la Lune, et leurs équations, ainsi que la différence entre la plus grande excentricité dans les syzygies et la plus petite dans les quadratures, et l'inégalité qu'on appelle variation ; toutes ces quantités augmentent et diminuent annuellement (par le Cor. 14 de la Prop. 66) en raison triplée du diamètre apparent du Soleil. De plus, la variation augmente ou diminue à peu près en raison doublée du temps qui s'écoule entre les quadratures (par les Cor. 1. et 2. du Lemme 10 et le Cor. 16 de la Prop. 66 Liv. 1) mais cette inégalité est ordinairement rapportée dans le calcul astronomique à la prosthaphérèse de la Lune, et est confondue avec elle.

\section{Proposition XXIII. - Problème V.}

Les inégalités des mouvements des satellites de Jupiter et de Saturne peuvent se déduire des mouvements de la Lune.

On peut déduire des mouvements de notre Lune les mouvements analogues des Lunes ou des satellites de Jupiter, et cela en cette sorte.

Par le Cor. 16. de la Prop. 66. du Liv. 1. le mouvement moyen des nœuds du satellite le plus éloigné de Jupiter est au mouvement moyen des nœuds de notre Lune, en raison composée de la raison doublée du temps périodique de la Terre autour du Soleil, au temps périodique de Jupiter autour du Soleil, et de la raison simple du temps périodique de ce satellite autour de Jupiter au temps périodique de la Lune autour de la Terre, ainsi en cent ans les nœuds du dernier satellite de Jupiter seront $8^{\circ} 24^{\prime}$ en antécédence.

Par le même corollaire, les mouvements moyens des nœuds des satellites intérieurs sont au mouvement des nœuds de ce dernier satellite comme les temps périodiques de ces satellites intérieurs au temps périodique du satellite extérieur, ainsi ils sont donnés. 
Il suit encore du même Corollaire que le mouvement en conséquence de l'apside la plus haute d'un satellite est au mouvement de ses nœuds en antécédence, comme le mouvement de l'apogée de notre Lune au mouvement de ses nœuds, et il est par conséquent donné.

Le mouvement de la plus haute apside ainsi trouvé, doit être diminué dans la raison de 5 à 9 ou de 1 à 2 à peu près, pour une raison qu'il n'est pas à propos d'expliquer ici.

Les plus grandes équations des nœuds, et de l'apside la plus haute d'un satellite quelconque sont, à peu près, aux plus grandes équations des nœuds et de l'apside la plus haute de la Lune, respectivement, comme le mouvement des nœuds et de l'apside la plus haute des satellites dans le temps d'une révolution des premières équations, au mouvement des nœuds et de l'apogée de la Lune dans le temps d'une révolution des dernières équations.

La variation d'un satellite, telle qu'on l'observerait de Jupiter, est à la variation de la Lune comme sont entre eux les mouvements entiers des nœuds pendant les temps pendant lesquels ce satellite et la Lune font leur révolution autour du Soleil, par le même Cor. Ainsi dans le satellite le plus éloigné de Jupiter elle ne passe pas 5 " $12 \mathrm{~m}$.

\section{Proposition XXIV. - THÉORÈME XIX.}

Le flux et reflux de la mer sont causés par les actions de la Lune et du Soleil.

Par les Cor. 19 et 20 de la Prop. 66 du premier Livre, on voit que la mer doit s'abaisser et s'élever deux fois chaque jour tant solaire que lunaire, et que la plus grande élévation de l'eau dans les mers libres et profondes, doit suivre le passage de l'astre par le méridien du lieu dans un espace de temps moindre que six heures. C'est en effet ce qui arrive dans la mer Atlantique et d'Éthiopie, et dans tout le trajet qui est entre la France et le Cap de Bonne-Espérance vers l'Orient, ainsi que dans la mer Pacifique sur les rivages du Chili et du Pérou : car dans toutes ces côtes les marées arrivent vers la 2, 3, ou quatrième heure, excepté que dans les lieux où l'eau rencontre beaucoup de sables, la marée retarde jusqu'à la 5, 6 et septième heure, et quelquefois au-delà. Je compte les heures depuis le passage de l'un et de l'autre 
astre par le méridien du lieu tant au-dessus qu'au-dessous de l'horizon, et par les heures du jour lunaire j'entends la vingtquatrième partie du temps que la Lune emploie dans son mouvement diurne apparent à revenir au méridien du lieu.

La plus grande force du Soleil ou de la Lune, pour élever les eaux de la mer, se trouve dans le moment même qu'ils atteignent le méridien du lieu. Cette force qu'ils impriment alors à la mer subsiste pendant un certain temps, et s'augmente par la force nouvelle qui lui est ensuite imprimée, jusqu'à ce que la mer soit parvenue à la plus grande hauteur, ce qui arrive dans l'espace d'une heure, de deux heures, et le plus souvent dans celui de trois heures environ vers les rivages, ou même dans un temps plus long, si la mer a beaucoup de bancs.

Les deux mouvements que ces deux astres excitent, ne peuvent pas être aperçus chacun à part, mais il s'en compose un mouvement mixte. Dans la conjonction ou l'opposition de ces astres, leurs actions conspirent et causent le plus grand flux et le plus grand reflux. Dans les quadratures, le Soleil élève l'eau lorsque la Lune l'abaisse, et il l'abaisse lorsque la Lune l'élève ; et la marée étant l'effet de la différence de ces actions opposées, elle est alors la plus petite. Or comme l'expérience fait voir que la Lune fait plus d'effet sur la mer que le Soleil, la plus grande hauteur de l'eau arrive, à peu près, à la troisième heure lunaire.

Hors des syzygies et des quadratures, la plus grande hauteur de l'eau devrait toujours arriver à la troisième heure lunaire par la seule action de la Lune, et à la troisième heure solaire par la seule action du Soleil ; et par ces actions composées elle arrive à un temps intermédiaire, mais qui est plus près de la troisième heure lunaire que de la troisième heure solaire ; ainsi dans le passage de la Lune des syzygies aux quadratures, où la troisième heure solaire précède la troisième heure lunaire, la plus grande hauteur de l'eau précède aussi la troisième heure lunaire, et elle la précède d'un intervalle qui est le plus grand un peu après les octants de la Lune; dans le passage des quadratures aux syzygies c'est le contraire, la plus haute marée suit la troisième heure lunaire avec des intervalles égaux à ceux avec lesquels elle l'avait précédée. 
Telles sont les lois du flux et du reflux dans les mers libres, mais aux embouchures des fleuves, les plus grandes hauteurs de l'eau arrivent plus tard, toutes choses d'ailleurs égales.

Les effets du Soleil et de la Lune sur la mer dépendent de leurs distances de la Terre ; car dans leurs moindres distances ils sont de plus grands effets, et dans leurs plus grandes distances leurs effets sont moindres, et cela en raison triplée de leurs diamètres apparents. Ainsi le Soleil étant l'hiver dans son périgée, il fait plus d'effet sur la mer, et par conséquent, toutes choses égales, les marées sont un peu plus hautes dans les syzygies, et un peu moindres dans les quadratures, en $\mathrm{Hi}-$ ver qu'en Été ; et la Lune étant chaque mois dans son périgée, les marées sont plus grandes alors que 15 jours devant ou 15 jours après qu'elle soit dans son apogée. Par ces deux causes il arrive que dans deux syzygies continues les deux plus grandes marées ne se suivent pas exactement.

Les effets du Soleil et de la Lune sur la mer dépendent aussi de la déclinaison de ces astres, ou de leur distance de l'équateur; car si l'astre était dans le pôle, il attirerait d'une manière constante toutes les parties de l'eau, sans que son action fut augmentée ni diminuée, et par conséquent elle n'exciterait aucun mouvement de réciprocité. Donc ces astres s'éloignant de l'équateur vers le pôle, leurs effets doivent diminuer peu à peu, et par conséquent ils doivent causer de moindres marées dans leurs syzygies solsticiales que dans leurs syzygies équinoxiales. Dans leurs quadratures solsticiales elles doivent, au contraire, être plus grandes que dans leurs quadratures équinoxiales; parce que les effets de la Lune, qui est alors dans l'équateur, surpassent beaucoup ceux du Soleil : ainsi les plus grandes marées arrivent dans les syzygies, et les moindres dans les quadratures de ces astres, vers les temps de l'équinoxe de l'un et de l'autre, et la plus grande marée dans les syzygies est toujours accompagnée de la plus petite dans les quadratures, comme l'expérience le fait voir.

Le Soleil étant moins éloigné de la Terre en Hiver qu'en Été, les plus grandes et les plus petites marées précèdent plus souvent l'équinoxe du Printemps qu'elles ne le suivent, et elles suivent plus souvent l'équinoxe d'Automne qu'elles ne le précèdent.

(Fig. 2) 
Les effets du Soleil et de la Lune sur la mer dépendent encore de la latitude des lieux. Que ApEP représente la Terre couverte de toutes parts par une mer très profonde ; que $C$ soit son centre ; $P$ et $p$ ses pôles; $A E$ son équateur; $F$ un lieu quelconque de la Terre pris hors de l'équateur; $F f$ le parallèle de ce lieu ; $D d$ le parallèle qui lui répond de l'autre côté de l'équateur; $L$ le lieu où la Lune était trois heures auparavant; $H$ le lieu de la Terre qui y répond perpendiculairement; $h$ le lieu opposé à celui-là ; $K, k$ les lieux qui en sont distants de $90^{\circ} ; \mathrm{CH}$, $C h$ les plus grandes hauteurs de la mer mesurées du centre de la Terre ; et $C K, C k$ ses plus petites hauteurs : si sur les axes $H h, K k$ on décrit une ellipse, cette ellipse par sa révolution autour de son grand axe Hh décrira un sphéroïde HPKhpk; lequel représentera à peu près la figure de la mer, et $C F, C f, C D$, $C d$ seront les hauteurs de la mer aux lieux $F, f, D$ et $d$. De plus, si dans la révolution de l'ellipse dont on vient de parler, un point quelconque $N$ décrit un cercle $M N$, lequel coupe les parallèles $F f$, $D d$ dans les lieux quelconques $R$ et $T$ et l'équateur $A E$ en $S$; $C N$ sera la hauteur de la mer dans tous les lieux $R, S, T$, situés dans ce cercle. Ainsi dans la révolution diurne d'un lieu quelconque $F$, l'élévation des eaux sera la plus grande en $F$, la troisième heure après le passage de la Lune par le méridien sur l'horizon ; et leur plus grand abaissement sera en $Q$ la troisième heure après le coucher de la Lune; ensuite la plus grande élévation sera en $f$ la troisième heure après le passage de la Lune par le méridien sous l'horizon; et enfin le plus grand abaissement en $Q$ la troisième heure après le lever de la Lune; et la dernière élévation des eaux en $f$ sera moindre que la première en $F$.

Supposons toute la mer séparée en deux flots hémisphériques, l'un boréal dans l'hémisphère $K H k$, et l'autre austral dans l'hémisphère opposé Khk; ces flots étant toujours opposés l'un à l'autre viennent tour à tour au méridien de chaque lieu de la Terre dans l'intervalle de 12 heures lunaires. Mais comme les régions boréales participent plus du flux boréal, et les australes du flux austral, il doit s'en composer des marées qui seront alternativement plus grandes et moindres dans chacun des lieux hors de l'équateur, dans lesquels le Soleil et la Lune se lèvent et se couchent. Ainsi la plus grande marée, lorsque la Lune décline vers le Zenith du lieu, tombera à peu près à la troisième heure après le passage de la Lune au méridien sur l'horizon ; et la déclinaison de la Lune changeant, cette plus grande marée deviendra la plus 
petite. La plus grande différence de ces marées tombera dans le temps des solstices; surtout si le nœud ascendant de la Lune se trouve dans le premier point d'Aries. C'est ce qui est conforme à l'expérience, car en Hiver les marées du matin sont plus grandes que celles du soir, et en Été celles du soir surpassent celles du matin. À Plymouth cette différence va presque à un pied, et à Bristol elle va à 15 pouces : comme l'ont observé Colepress et Sturmius.

Les mouvements de la mer dont j'ai parlé jusqu'à présent sont un peu altérés par cette force de réciprocité des eaux, par laquelle le flux pourrait subsister quelque temps quoique les actions du Soleil et de la Lune sur la mer vinssent à cesser. Cette conservation du mouvement une fois imprimé diminue la différence des marées alternatives; et elle rend les marées plus grandes immédiatement après les syzygies, et plus petites immédiatement après les quadratures. C'est pourquoi les marées alternatives à Plymouth et à Bristol ne diffèrent pas entre elles beaucoup plus que d'un pied ou de 15 pouces; en sorte que les plus grandes marées dans ces ports ne sont pas les premières après les syzygies, mais les troisièmes.

Tous ces mouvements sont retardés lorsque les eaux de la mer passent sur des bas fonds, ainsi les plus grandes marées dans les détroits et dans les embouchures des fleuves, ne sont que le quatrième ou même le cinquième jour après les syzygies.

De plus, il peut se faire que le flux se propage de l'océan par plusieurs détroits jusqu'au même port, et qu'il passe plus vite par quelques-uns de ces détroits que par les autres : d'où il arrive que le même flux étant divisé en deux ou plusieurs flux qui arrivent successivement, il peut composer de nouveaux mouvements de différents genres. Supposons deux flux égaux qui arrivent de deux endroits différents dans le même port, et dont l'un précède l'autre de six heures, et tombe dans la troisième heure après le passage de la Lune par le méridien de ce port; si la Lune, lorsqu'elle arrive à ce méridien, était dans l'équateur, il y aurait toutes les six heures des flux qui seraient contrebalancés par des reflux égaux et l'eau serait stagnante pendant tout l'espace de ce jour-là ; mais si la Lune déclinait alors, les marées seraient tour à tour plus grandes et moindres dans l'océan, comme on l'a dit ; et elles se propageraient de l'océan dans ce port deux à deux ; ainsi il y arriverait deux marées fortes et deux marées faibles tour à 
tour. Les deux marées fortes feraient que l'eau acquerrait sa plus grande hauteur dans le milieu entre l'une et l'autre, la marée forte et la marée faible feraient que l'eau acquerrait sa hauteur moyenne entre ces deux marées, et entre les deux marées faibles l'eau monterait à sa moindre hauteur. Ainsi dans l'espace de 24 heures l'eau n'acquerrait pas deux fois, comme il arrive ordinairement, mais seulement une fois sa plus grande hauteur, et une fois sa moindre hauteur. La plus grande hauteur de l'eau, si la Lune décline vers le pôle qui est sur l'horizon du lieu, tombera à la sixième ou à la treizième heure après le passage de la Lune au méridien, et elle se changera en reflux lorsque la déclinaison de la Lune changera.

Halley a trouvé des exemples de tout cela dans les observations des pilotes faites à Batsham port du royaume de Tonquin, situé à $20^{\circ} 50^{\prime}$ de latitude boréale. Dans ce port, il n'y a point de marée le jour qui suit le passage de la Lune par l'équateur, ensuite, lorsque la Lune commence à décliner vers le Nord on commence à s'apercevoir du flux et du reflux, non pas deux fois par jour comme dans les autres ports, mais une fois seulement chaque jour; et le flux arrive lorsque la Lune se couche, et le reflux lorsqu'elle se lève.

Le flux augmente dans ce port avec la déclinaison de la Lune jusqu'au septième ou huitième jour, ensuite il diminue par les mêmes degrés pendant sept autres jours, et lorsqu'ensuite la Lune passe dans les signes opposées il cesse entièrement et se change après en reflux. Le reflux arrive alors au coucher de la Lune, et le flux à son lever, jusqu'à ce que la Lune revienne dans les premiers signes.

On arrive à ce port par deux détroits, l'un qui est dans la mer de la Chine entre le continent et l'île de Laconie, l'autre dans la mer des Indes entre le continent et l'île de Bornéo. De savoir si les marées, en passant par ces détroits, et venant de la mer des Indes dans l'espace de 12 heures, et de la mer de la Chine dans l'espace de 6 heures, et en arrivant ainsi à la troisième et à la neuvième heure lunaire, composent seules ces forces de mouvements, ou s'il ne s'y mêle point d'autres causes propres à ces mers, c'est ce que je laisse à déterminer par les observations qu'on pourra faire sur les côtes voisines. 
J'ai expliqué jusqu'ici les causes des mouvements de la Lune et de la mer, il me reste à traiter à présent de la quantité de ces mouvements.

\section{Proposition XXV. - Problème VI.}

Trouver les forces du Soleil pour troubler les mouvements de la Lune.

(Fig. 3)

Que $S$ représente le Soleil, $T$ la Terre, $P$ la Lune, $C A D B$ l'orbe de la Lune. Que $S K$ prise sur $S P$ soit égale à $S T$; et que $S L$ soit à $S K$ en raison doublée de $S K$ à $S P$; enfin que $L M$ soit parallèle à $P T$; si la gravité accélératrice de la Terre vers le Soleil est exprimée par la distance $S T$ ou $S K, S L$ sera la gravité accélératrice de la Lune vers le Soleil, laquelle est composée des parties $S M, L M$, desquelles $L M$ et la partie $T M$ de $S M$ troublent les mouvements de la Lune, comme on l'a fait voir au Livre premier dans la Proposition 66. et ses Corollaires.

La Terre et la Lune faisant leur révolution autour de leur commun centre de gravité, le mouvement de la Terre autour de ce centre est aussi troublé par des forces semblables; mais on peut rapporter la somme de ces mouvements et de ces forces à la Lune et représenter les sommes de ces forces par des lignes analogues TM et $L M$.

La force $L M$, dans sa moyenne quantité, est à la force centripète, par laquelle la Lune peut faire sa révolution dans son orbite à la distance $P T$, autour de la Terre supposée en repos, en raison doublée des temps périodiques de la Lune autour de la Terre et de la Terre autour du Soleil, par le Cor. 17. de la Prop. 66. du Liv. I. c'est-à-dire, en raison doublée de 27 jours, $7^{\mathrm{h}} 43^{\prime}$ à 365 jours $6^{\mathrm{h}} 9^{\prime}$, ou, ce qui revient au même, comme 1000 à 178 725, ou enfin comme 1 à $178 \frac{29}{40}$. Or nous avons trouvé dans la Prop. 4. que si la Terre et la Lune tournent autour d'un commun centre de gravité, leur moyenne distance entre elles sera environ de $60 \frac{1}{2}$ demi-diamètres médiocres de la Terre à peu près; et la force par laquelle la Lune peut tourner dans son orbe autour de la Terre en repos, à la distance $P T$, qui est de $60 \frac{1}{2}$ demi-diamètres de la Terre, est à la force par laquelle elle peut y tourner dans le même temps à la distance de 60 demi-diamètres comme $60 \frac{1}{2}$ est à 60 ; de 
plus, cette force est à la force de la gravité sur la Terre comme 1 à 60 $\times 60$ à peu près. Donc la force moyenne $M L$ est à la force de la gravité sur la surface de la Terre, comme $1 \times 60 \frac{1}{2}$ à $60 \times 60 \times 60 \times 178 \frac{29}{40}$, ou comme 1 à 638092,6 . Il n'est plus question maintenant que de connaître la proportion des lignes $T M, M L$ pour avoir la force $T M$, et par conséquent celles par lesquelles le Soleil trouble les mouvements de la Lune. - C.Q.F.T.

\section{Proposition XXVI. - Problème VII.}

Trouver l'incrément horaire de l'aire que la Lune décrit autour de la Terre, en supposant que son orbite soit circulaire.

Nous avons dit que les aires que la Lune décrit autour de la Terre sont proportionnelles au temps lorsqu'on néglige l'altération que l'action du Soleil cause dans les mouvements lunaires. Examinons ici quelle est l'inégalité du moment, ou de l'incrément horaire causée par cette action.

Afin de rendre le calcul plus facile, supposons l'orbe de la Lune parfaitement circulaire, et négligeons toutes ses inégalités, excepté celle dont il est ici question.

(Fig. 4)

À cause du grand éloignement du Soleil, supposons que les lignes $S P, S T$ soient parallèles entre elles; par ce moyen, la force $L M$ sera toujours réduite à sa moyenne quantité $T P$, ainsi que la force $T M$ à sa moyenne quantité $3 P K$. Ces forces, par le Cor. 2. des Lois, composent la force $T L$; laquelle, en abaissant $L E$ perpendiculairement sur le rayon $T P$, se résout dans les forces $T E, E L$, dont la première $T E$, agissant toujours selon le rayon $T P$, n'accélère ni ne retarde la description de l'aire TPC parcourue par le rayon TP; quant à la seconde EL, comme elle agit selon la perpendiculaire à ce rayon, elle accélère ou retarde cette description autant qu'elle retarde ou accélère le mouvement de la Lune. Cette accélération de la Lune, qui se fait à chaque instant, dans son passage de la quadrature $C$ à la conjonction $A$, est comme la force même accélérante $E L$, c'est-à-dire, comme $\frac{3 P K \times T K}{T P}$.

(Fig. 4) 
Que le temps soit représenté par le moyen mouvement de la Lune ou (ce qui revient presque au même) par l'angle CTP, ou encore par l'arc $C P$. Qu'on tire $C G$ perpendiculaire et égale à $C T$; et qu'on suppose le quart de cercle $A C$ divisé en un nombre infini de petites parties égales $P p$, etc. qui représentent autant de petites parties égales de temps, qu'on mène de plus $p k$ perpendiculaire à $C T$, et qu'on tire $T G$ qui rencontre en $F$ et en $f$ ces mêmes lignes $K P, k p$ prolongées; il est clair que $F K$ sera égale à $T K$, et qu'on aura $K k: P K=P p: T p$, c'est-àdire, en raison donnée ; donc $F K \times K k$ ou l'aire $F K k f$ sera comme $\frac{3 P K \times T K}{T P}$; c'est-à-dire, comme $E L$; et par conséquent l'aire totale GCKF sera comme la somme de toutes les forces $E L$ imprimées à la Lune pendant tout le temps $C P$, et par conséquent comme la vitesse que toutes ces forces ont produite, c'est-à-dire, comme l'accélération de la description de l'aire CTP ou comme l'incrément du moment.

La force par laquelle la Lune peut faire sa révolution autour de la Terre, supposée en repos, à la distance $T P$, dans le temps périodique $C A D B$ de 27 jours, $7^{\mathrm{h}} 43^{\prime}$, ferait qu'un corps en tombant pendant le temps $C T$ parcourrait la longueur $\frac{1}{2} C T$, et acquerrait en même temps une vitesse égale à celle de la Lune dans son orbe ; ce qui est clair par le Cor. 9. de la Prop. 4, Liv. 1. Or comme la perpendiculaire $K d$ abaissée sur TP est la troisième partie de EL, et la moitié de TP ou de $M L$ dans les octants, la force $E L$ dans les octants, où elle est la plus grande, surpassera la force $M L$ dans la raison de 3 à 2, ainsi elle sera à la force par laquelle la Lune peut tourner autour de la Terre en repos, dans son temps périodique, comme 100 à $\frac{2}{3} \times 17872 \frac{1}{2}$ ou 11915 , et dans le temps CT elle devrait produire une vitesse qui serait la $\frac{100}{11915}$ partie de la vitesse de la Lune, et pendant le temps CPA elle devrait produire une vitesse qui serait plus grande dans la raison de $C A$ à $C T$ ou $T P$.

Que la plus grande force $E L$ dans les octants soit représentée par l'aire $F K \times K k$ égale au rectangle $\frac{1}{2} T P \times P p$. La vitesse que la plus grande force peut produire dans un temps quelconque $C P$ sera à la vitesse que la plus petite force entière $E L$ peut produire dans le même temps, comme le rectangle $\frac{1}{2} T P \times C P$ à l'aire $K C G F$ : et les vitesses produites pendant le temps total CPA seront entre elles comme le rectangle $\frac{1}{2} T P \times C A$ et le triangle $T C G$, ou comme l'arc d'un quart de 
cercle $C A$ et son rayon $T P$. Donc la vitesse à la fin du temps total sera la $\frac{100}{11915}$ partie de la vitesse de la Lune. Si l'on ajoute, et si l'on ôte de cette vitesse de la Lune, qui est proportionnelle à l'incrément médiocre de l'aire, la moitié de cette dernière vitesse, et qu'on représente l'incrément moyen par le nombre 11915 , la somme $11915+50$ ou 11965 représentera le plus grand incrément de l'aire dans la syzygie $A$, et la différence 11915 - 50 ou 11865 le plus petit incrément de cette même aire dans les quadratures. Donc les aires décrites en temps égaux dans les syzygies et dans les quadratures sont entre elles, comme 11965 et 11865 . Ajoutant au plus petit incrément 11865, un incrément qui soit à la différence 100 des incréments, comme le trapèze FKCG au triangle TCG, ou (ce qui est la même chose) comme le carré du sinus $P K$ au carré du rayon $T P$ c'est-à-dire, comme $P d$ à $T P$, la somme représentera l'incrément de l'aire, lorsque la Lune se trouve dans un lieu intermédiaire quelconque $P$.

Tout cela a lieu dans l'hypothèse que le Soleil et la Terre soient en repos, et que la Lune fasse sa révolution dans le temps synodique de 27 jours, $7^{\mathrm{h}} 43^{\mathrm{\prime}}$. Mais comme la vraie période synodique lunaire est de 29 jours, $12^{\mathrm{h}} 44^{\prime}$, les incréments des moments doivent augmenter en raison du temps, c'est-à-dire, en raison de 1080853 à 1000 000. De cette manière, l'incrément total, qui était la $\frac{10}{11915}$ partie du moment médiocre, deviendra sa $\frac{100}{11025}$ partie. Ainsi le moment de l'aire dans la quadrature de la Lune sera au moment de cette même aire dans la syzygie, comme 11023 - 50 à $11023+50$, ou comme 10973 à 11073 ; et à son moment, lorsque la Lune est dans un lieu quelconque intermédiaire $P$, comme 10973 à $10973+P d$, en supposant $T P=100$.

Donc l'aire que la Lune décrit autour de la Terre à chaque particule égale de temps, est à peu près comme la somme du nombre 219,46 et du sinus verse du double de la distance de la Lune à la prochaine quadrature, dans un cercle dont le rayon est l'unité. Tout ceci suppose que la variation dans les octants soit de grandeur médiocre. Si la variation y est plus grande ou plus petite, ce sinus verse doit être augmenté ou diminué dans la même raison. 


\section{Proposition XXVII. — ProblèME VIII.}

Par le mouvement horaire de la Lune trouver quelle est sa distance de la Terre.

L'aire que la Lune décrit à chaque moment autour de la Terre, est comme le mouvement horaire de la Lune, et le carré de la distance de la Lune à la Terre conjointement; et par conséquent, la distance de la Lune à la Terre est en raison composée de la raison sousdoublée de l'aire directement, et de la raison sousdoublée inverse du mouvement horaire. - C.Q.F.T.

Cor. 1. On a, par ce moyen, le diamètre apparent de la Lune car il est réciproquement comme sa distance à la Terre. C'est aux Astronomes à voir combien cette règle s'accorde exactement avec les Phénomènes.

Cor. 2. On peut encore tirer de là un moyen d'employer les Phénomènes à déterminer l'orbite de la Lune beaucoup plus exactement qu'on n'a fait jusqu'à présent.

\section{Proposition XXVIII. - Problème IX.}

Trouver les diamètres de l'orbe dans lequel la Lune devrait se mouvoir, en supposant quelle n'eût point d'excentricité.

La courbure de la trajectoire qu'un mobile décrirait s'il était toujours tiré perpendiculairement à cette trajectoire, est en raison directe de l'attraction, et en raison inverse du carré de la vitesse. Je suppose que les courbures des courbes sont entre elles dans la dernière proportion des sinus, ou des tangentes des angles de contact qui appartiennent aux rayons égaux, lorsque ces rayons diminuent à l'infini.

(Fig. 3)

L'attraction de la Lune vers la Terre dans les syzygies est l'excès de sa gravité vers la Terre sur la force solaire $2 P K$, laquelle est la différence des gravités de la Lune et de la Terre vers le Soleil : et dans les quadratures, cette attraction est la somme de la gravité de la Lune vers la Terre, et de la force solaire $K T$ dirigée vers la Terre. Ces attractions, en nommant $N$ la quantité $\frac{A T+C T}{2}$ sont, à peu près, comme 
$\frac{178725}{A T^{2}}-\frac{2000}{C T \times N}$ et $\frac{178725}{C T^{2}}+\frac{1000}{A T \times N}$; ou comme $178725 N \times C T^{2}$ $-2000 A T^{2} \times C T$ et $178725 N \times A T^{2}+1000 C T^{2} \times A T$. Car si la gravité accélératrice de la Lune vers la Terre est représentée par le nombre 178725 , la force médiocre $M L$, qui dans les quadratures est $P T$ ou $T K$, et qui tire la Lune vers la Terre, sera 1000, et la force médiocre $T M$ dans les syzygies sera 3000 ; de laquelle, si on ôte la force médiocre $M L$, il restera la force 2000, par laquelle la Lune s'éloigne de la Terre dans les syzygies, et laquelle j'ai nommé ci-devant $2 P K$.

La vitesse de la Lune dans les syzygies $A$ et $B$ est à la vitesse dans les quadratures $C$ et $D$, comme $C T$ à $A T$, et comme le moment de l'aire que la Lune décrit dans les syzygies autour de la Terre, est au moment de cette même aire dans les quadratures conjointement, c'està-dire, comme 11073CT à 10973AT.

Cela posé, il est évident que la courbure de l'orbe de la Lune dans les syzygies est à sa courbure dans les quadratures comme 120406729 $\times 178725 A T^{2} \times C T^{2} \times N-120406729 \times 2000 A T^{4} \times C T$ à $122611329 \times 178725 A T^{2} \times C T^{2} \times N+122611329 \times 1000 C T^{4} \times A T$, c'est-à-dire, comme $2151969 A T \times C T \times N-24081 A T^{3}$ à $2191371 A T$ $\times C T \times N+12261 C T^{3}$.

(Fig. 5)

Comme on ignore la figure de l'orbe de la Lune, nous supposerons que cet orbe fait l'ellipse $D B C A$ dans le centre $T$ de laquelle la Terre est placée, et dont le grand axe $D C$ passe par les quadratures, et le petit axe $A B$ par les syzygies. Et à cause du fait que le plan de cette ellipse se meut d'un mouvement angulaire autour de la Terre, et que la trajectoire dont nous cherchons la courbure doit être décrite dans un plan qui soit entièrement privé de tout mouvement angulaire : il faut considérer la figure que la Lune, en faisant sa révolution dans cette ellipse, décrit dans ce plan immobile, c'est-à-dire, la figure Cpa, dont chaque point $p$ est déterminé en prenant un point quelconque $P$ dans l'ellipse pour représenter le lieu de la Lune, et en menant Tp égale à $T P$, par une loi telle que l'angle PTp soit égal au mouvement apparent du Soleil depuis la quadrature $C$; ou (ce qui revient à peu près au même) que l'angle CTp soit à l'angle CTP comme le temps de la révolution synodique de la Lune est au temps de sa révolution périodique, ou comme 29 jours $12^{\mathrm{h}} 44^{\prime}$ à 27 jours $7^{\mathrm{h}} 43^{\prime}$. 
(Fig. 5)

Prenant donc l'angle $C T a$ dans cette raison à l'angle droit CTA, et faisant $T a$ égale à $T A$; $a$ sera l'apside la plus basse, et $C$ l'apside la plus haute de cet orbe Cpa quant aux courbures dans ces deux points, je trouve, en faisant le calcul nécessaire, que la différence entre la courbure de l'orbe Cpa au sommet $a$, et la courbure du cercle dont le centre est $T$ et le rayon $T A$ est à la différence entre la courbure de l'ellipse au sommet $A$, et la courbure de ce même cercle, en raison doublée de l'angle CTP à l'angle CTp; et que la courbure de l'ellipse en $A$ est à la courbure de ce cercle, en raison doublée de $T A$ à $T C$; de plus, que la courbure de ce cercle est à la courbure du cercle dont le centre est $T$ et le rayon $T C$ comme $T C$ à $T A$; et que cette courbure est à la courbure de l'ellipse en $C$, en raison doublée de $T A$ à $T C$; et enfin que la différence entre la courbure de l'ellipse au sommet $C$ et la courbure de ce dernier cercle, est à la différence entre la courbure de la figure Tpa au sommet $C$, et la courbure de ce même cercle, en raison doublée de l'angle CTp à l'angle CTP. Ce qui se tire aisément des sinus des angles de contact, et des différences de ces angles.

Employant donc toutes ces raisons, on trouve que la courbure de la figure Cpa en $a$, est à sa courbure en $C$, comme $A T^{3}+\frac{16824}{100000} C T^{2} \times A T$ à $C T^{3}+\frac{16824}{100000} A T^{2} \times C T$. Le nombre $\frac{16824}{100000}$ représentant la différence des carrés des angles CTP et CTp divisée par le carré du plus petit angle CTP ou, ce qui est la même chose, la différence des carrés des temps 27 jours $7^{\mathrm{h}} 43^{\prime}$ et 29 jours $12^{\mathrm{h}} 44^{\prime}$ divisée par le carré du temps 27 jours $7^{\text {h }} 43^{\prime}$.

Donc puisque $a$ représente la syzygie de la Lune, et $C$ sa quadrature, la proportion qu'on vient de trouver doit être la même que celle de la courbure de l'orbe de la Lune dans les syzygies à la courbure du même orbe dans les quadratures, qui a été trouvée ci-dessus. C'est pourquoi, pour trouver la proportion de $C T$ à $A T$, il n'y a qu'à multiplier les extrêmes et les moyens entre eux ; et les termes qui en viendront étant divisés par TC $\times A T$ donneront l'équation $2062,79 C T^{4}-$ $2151969 N \times C T^{3}+368676 N \times A T \times C T^{2}+36342 A T^{2} \times C T^{2}-$ $362047 N \times A T^{2} \times C T+2191371 N \times A T^{3}+4051,4 A T^{4}=0$. Dans laquelle, si au lieu de la demi-somme $N$ des termes $A T, C T$, on met 1 , et au lieu de leur demi-différence $x$, et par conséquent $1+x$ au lieu de $C T$, et $1-x$ au lieu de $A T$; on aura $x=0,00719$, c'est-à-dire, que le 
demi-diamètre $C T$ sera 1,00719, et le demi-diamètre AT 0,99281 : lesquels nombres sont entre eux à peu près comme $70 \frac{1}{24}$ et $69 \frac{1}{24}$. La distance de la Lune à la Terre dans les syzygies, est donc à sa distance dans les quadratures comme $69 \frac{1}{24}$ à $70 \frac{1}{24}$, ou en nombres ronds comme 69 à 70, pourvu qu'on fasse abstraction de l'excentricité.

\section{Proposition XXIX. - Problème X.}

\section{Trouver la variation de la Lune.}

(Fig. 5) Cette inégalité de la Lune vient en partie de l'inégalité des moments de l'aire que la Lune décrit autour de la Terre, et en partie de la forme elliptique de l'orbe lunaire. Supposant que la Lune se meuve dans une ellipse DBCA autour de la Terre en repos, placée dans le centre de cette ellipse, elle décrira des aires CTP proportionnelles aux temps; et si le demi grand diamètre $C T$ de l'ellipse est à son petit demi-diamètre $T A$ comme 70 à 69 , la tangente de l'angle CTP sera à la tangente de l'angle du mouvement moyen calculé depuis la quadrature $C$, comme 69 à 70. Mais la description de l'aire CTP, lorsque la Lune passe de la quadrature à la syzygie, doit être accélérée, en telle sorte que son moment dans la syzygie soit à son moment dans la quadrature comme 11073 à 10973, et que l'excès du moment dans un lieu intermédiaire quelconque $P$, sur le moment dans la quadrature, soit comme le carré du sinus de l'angle CTP. C'est ce qu'on fera assez exactement, si on diminue la tangente de l'angle CTP en raison sousdoublée du nombre 10973 au nombre 11073, c'est-à-dire, en raison du nombre 68,6877 au nombre 69. Par ce moyen, la tangente de l'angle CTP sera à la tangente du mouvement moyen comme 68,6877 à 70. Et l'angle CTP dans les octants, où le mouvement moyen est de $45^{\circ}$, sera de $44^{\circ} 27^{\prime} 28^{\prime \prime}$, qui étant ôté de l'angle du mouvement moyen qui est de $45^{\circ}$ donnera $32^{\prime} 32^{\prime \prime}$ pour la plus grande variation.

Ce serait là la plus grande variation, si la Lune, en passant de la quadrature à la syzygie, décrivait un angle CTA qui fut exactement de 90 degrés. Mais à cause du mouvement de la Terre, par lequel le Soleil avance en conséquence par son mouvement apparent, la Lune, avant d'avoir atteint le Soleil, décrit un angle $C T a$, qui est plus grand qu'un angle droit, dans la raison du temps de la révolution synodique de la Lune au temps de sa révolution périodique, c'est-à-dire, en rai- 
son de 29 jours $12^{\mathrm{h}} 44^{\prime}$ à 27 jours, $7^{\mathrm{h}} 43^{\prime}$. Il faut donc augmenter tous les angles autour du centre $T$ dans la même raison, ce qui au lieu de $32^{\prime} 32^{\prime \prime}$ pour la plus grande variation donnera $35^{\prime} 10^{\prime \prime}$.

C'est là la grandeur de la variation dans la moyenne distance du Soleil à la Terre, en négligeant les différences qui peuvent naître de la courbure du grand orbe, et de la quantité dont l'action du Soleil sur la Lune, lorsqu'elle est nouvelle et en croissant surpasse l'action de ce même astre sur la Lune lorsqu'elle est pleine et gibbeuse.

Dans les autres distances du Soleil à la Terre, la plus grande variation est en raison composée de la raison doublée directe du temps de la révolution synodique de la Lune (pour le temps donné de l'année) et de la raison inverse triplée de la distance du Soleil à la Terre. Ainsi dans l'apogée du Soleil, la plus grande variation est de 33' 14", et dans son périgée, elle est de 37' 11", supposé que l'excentricité du Soleil soit au demi-diamètre transversal du grand orbe comme $16 \frac{15}{16}$ à 1000.

Nous avons trouvé jusqu'à présent la variation de la Lune en supposant que son orbe ne soit point excentrique, et que lorsqu'elle est dans ses octants elle soit toujours à sa médiocre distance de la Terre. Mais comme la Lune par son excentricité est tantôt plus près et tantôt plus loin de la Terre qu'elle ne l'est dans l'orbe qu'on vient d'examiner, sa variation pourra être un peu plus grande, ou un peu moindre que la précédente : j'en laisse l'excès ou le défaut à déterminer aux astronomes par les Phénomènes.

\section{Proposition XXX. - Problème XI.}

Trouver le mouvement horaire des nœuds de la Lune dans un orbe circulaire.

(Fig. 6)

Que $S$ désigne le Soleil, $T$ la Terre, $P$ la Lune, $N P n$ l'orbe de la Lune, $N p n$ la projection de cet orbe dans le plan de l'écliptique; $N$ et $n$ les nœuds, $n T N m$ la ligne de ces nœuds prolongée infiniment, PI, $P K$ des perpendiculaires abaissées sur les lignes $S T, Q q ; P p$ une perpendiculaire abaissée sur le plan de l'écliptique ; $A$ et $B$ les syzygies de la Lune dans ce plan; $A Z$ une perpendiculaire à la ligne des nœuds 
$N n ; Q$ et $q$ les quadratures de la Lune dans le plan de l'écliptique, et $p K$ une perpendiculaire à la ligne $Q q$ des quadratures.

La force du Soleil pour troubler les mouvements de la Lune est composée de deux forces (par la Prop. 25) l'une proportionnelle à la ligne $L M$ de la figure de cette Proposition, et l'autre à la ligne $M T$ de la même figure. La Lune par la première de ces forces est tirée vers la Terre, et par la seconde vers le Soleil, suivant une ligne parallèle à la droite ST menée du Soleil à la Terre.

La première force $L M$ agissant dans le plan de l'orbite lunaire ne saurait altérer la situation de ce plan, ainsi elle ne doit point être considérée. Quant à la force $M T$ par laquelle le plan de l'orbite lunaire est dérangé, elle a pour expression $3 P K$ ou $3 I T$. Et cette force (par la Prop. 25.) est à celle par laquelle la Lune pourrait être mue uniformément (dans son temps périodique) dans un cercle autour de la Terre supposée fixe, comme $3 I T$ au rayon du cercle multiplié par le nombre 178,725, ou comme IT au rayon multiplié par 59,575. Au reste dans ce calcul et dans tout ce qui suit, je considère toutes les lignes menées de la Lune au Soleil comme parallèles à celles qui sont tirées de la Terre au Soleil, parce que l'inclinaison de ces lignes diminue à peu près tous les effets dans quelques cas, de la même manière qu'elle les augmente dans d'autres; et que nous cherchons les mouvements médiocres des nœuds, en négligeant les fractions insensibles qui rendraient le calcul trop embarrassant.

$P M$ désignant maintenant l'arc que la Lune décrit dans un instant donné, et $M L$ la petite ligne dont la Lune parcourrait la moitié dans le même temps en vertu de la force précédente $3 I T$; soient tirées $P L, P M$ que l'on prolonge en $m$ et en $l$, jusqu'à ce qu'elles rencontrent le plan de l'écliptique, et soit abaissée la perpendiculaire $P H$ de $P$ sur $T m$.

Parce que la droite $M L$ est parallèle au plan de l'écliptique, et que par conséquent elle ne peut rencontrer la droite $m l$ qui est dans ce plan, que de plus ces droites $M L, m l$, sont dans un même plan $L M P m l$; il faudra qu'elles soient parallèles, et par conséquent que les triangles $L M P, \operatorname{lm} P$ soient semblables.

Présentement, comme $M P m$ est dans le plan de l'orbite dans lequel la Lune se meut en $P$, le point $m$ tombera sur la ligne $N n$ menée par les nœuds $N, n$ de cette orbite : et parce que la force qui fait décrire la 
moitié de la petite ligne $L M$, ferait décrire cette ligne entière si elle était imprimée en une seule fois dans le lieu $P$; et qu'elle ferait mouvoir la Lune dans l'arc dont la corde serait $L P$, et transporterait par conséquent la Lune du plan MPmT dans le plan LPIT; le mouvement angulaire des nœuds engendré par cette force sera égal à l'angle $m T l$. Mais $m l: m P=M L: M P$, donc, à cause que $M P$ est donnée par la supposition du temps constant, $m l$ sera comme le rectangle $M L \times m P$, c'est-à-dire, comme le rectangle $I T \times m P$. Et l'angle $m T l$, si on suppose l'angle $\mathrm{Tml}$ droit, sera comme $\frac{\mathrm{ml}}{\mathrm{Tm}}$, et par conséquent comme $\frac{I T \times m P}{T m}$, ou ce qui revient au même, (à cause des proportionnelles $T m$ et $m P, T P$ et $P H)$ comme $\frac{I T \times P H}{T P}$ ou comme $I T \times P H$ à cause que $T P$ est donnée.

Mais comme l'angle Tml ou STN n'est pas droit, l'angle $m T l$ sera moindre, et cela dans la raison du sinus de l'angle $S T N$ au rayon, ou de $A Z$, à $A T$ Donc la vitesse des nœuds est comme $I T \times P H \times A Z$, c'est-à-dire, comme le produit des sinus des trois angles TPI, PTN et STN.

Si ces angles, les nœuds étant dans les quadratures, et la Lune dans la syzygie, sont droits, la petite droite $\mathrm{ml}$ se trouvera à une distance infinie, et l'angle $m T l$ deviendra égal à l'angle $m P l$. Or dans ce cas, l'angle $\mathrm{mPl}$ est à l'angle PTM que la Lune décrit dans le même temps par son mouvement apparent autour de la Terre, comme 1 à 59,575. Car l'angle $m P l$ est égal à l'angle $L P M$, c'est-à-dire, à l'angle de la déflexion de la Lune du chemin rectiligne, qui serait produite par la seule force solaire 3IT dans ce temps donné, si la Lune cessait d'être pesante ; de plus, l'angle PTM est égal à l'angle de la déflexion de la Lune du chemin rectiligne causée par la seule force qui la retient dans son orbite, en faisant abstraction de la force solaire 3IT. Et ces forces, comme nous l'avons dit ci-dessus, sont entre elles comme 1 à 59,575. Donc, comme le mouvement moyen horaire de la Lune, à l'égard des fixes, est de $32^{\prime} 56^{\prime \prime} 27^{\prime \prime \prime} 12^{\text {iv }} \frac{1}{2}$, le mouvement horaire du nœud sera, dans ce cas, de $33^{\prime \prime} 10^{\prime \prime \prime} 33^{\text {iv }} 12^{\mathrm{v}}$; et dans les autres cas, ce mouvement horaire sera à $33^{\prime \prime} 10^{\prime \prime \prime} 33^{\text {iv }} 12^{\mathrm{v}}$, comme le produit des sinus des trois angles TPI, PTN, et STN, (c'est-à-dire, de la distance de la Lune à la 
quadrature, de la distance de la Lune au nœud, et de la distance du nœud au Soleil) est au cube du rayon. Et toutes les fois que le signe d'un de ces angles passera du positif au négatif, et du négatif au positif, le mouvement des nœuds se changera de régressif en progressif, et de progressif en régressif. D'où il arrive que les nœuds avancent toutes les fois que la Lune est entre une des quadratures et le nœud le plus proche de la quadrature. Dans les autres cas, les nœuds rétrogradent, et en vertu de l'excès du mouvement rétrograde sur le mouvement progressif les nœuds seront portés chaque mois en antécédence.

(Fig. 7)

Cor. 1. De là il suit, que si on abaisse des extrémités $P$ et $M$ d'un arc donné infiniment petit $P M$, les perpendiculaires $P K, M k$ à la ligne $Q q$ qui passe par les quadratures, et qu'on prolonge ces perpendiculaires jusqu'à ce qu'elles coupent la ligne des nœuds $N n$ en $D$ et en $d$ le mouvement horaire des nœuds sera comme l'aire MPDd et le carré de la ligne $A Z$ conjointement. Car soient $P K, P H$ et $A Z$ les trois sinus dont on vient de parler, $P K$ étant le sinus de la distance de la Lune à la quadrature, $P H$ le sinus de la distance de la Lune au nœud, et $A Z$ le sinus de la distance du nœud au Soleil : on aura pour la vitesse du nœud le produit $P K \times P H \times A Z$. Mais $P T: P K=P M: K k$; donc, à cause des données $P T$ et $P M$, la petite droite $K k$ sera proportionnelle à $P K$. De plus, $A T: P D=A Z: P H$, et par conséquent $P H$ est proportionnelle à $P D \times A Z$. Donc $P K \times P H$ est comme $K k \times P D \times A Z$, et $P K \times P H \times A Z$ sera comme $K k \times P D \times A Z^{2}$, c'est-à-dire, comme l'aire $P D d M$ et $A Z^{2}$ conjointement. - C.Q.F.D.

Cor. 2. Dans une position quelconque donnée des nœuds, le mouvement horaire médiocre est la moitié du mouvement horaire dans les syzygies de la Lune, c'est-à-dire, que ce mouvement est à $16^{\prime \prime} 35^{\prime \prime \prime}$ $16^{\mathrm{iv}} 36^{\mathrm{v}}$, comme le carré du sinus de la distance des nœuds aux syzygies est au carré du rayon, ou ce qui revient au même, comme $A Z^{2}$ à $A T^{2}$.

(Fig. 7)

Car si la Lune parcourt d'un mouvement uniforme le demi-cercle $Q A q$, la somme de toutes les aires $P D d M$ décrites pendant le temps que la Lune va de $Q$ à $M$ sera l'aire $Q M d E$ terminée par la tangente $Q E$ du cercle; et la somme de toutes les aires $P D d M$ pendant que la Lune va en $n$ sera l'aire totale EQAn que la ligne $P D$ décrit, ensuite la 
Lune allant de $n$ en $q$, la ligne $P D$ tombera hors du cercle, et décrira l'aire nqe terminée par la tangente qe du cercle, laquelle aire, à cause $\mathrm{du}$ fait que les nœuds allaient d'abord en rétrogradant et vont alors en avançant, doit être retranchée de la première aire, et par son égalité à l'aire $Q E N$, le reste deviendra, le demi-cercle NQAn. Donc la somme de toutes les aires $P D d M$ décrites pendant le temps que la Lune parcourt un demi-cercle, est l'aire du demi-cercle; et la somme de toutes les mêmes aires décrites pendant le temps que la Lune parcourt le cercle entier, est l'aire du cercle entier.

Mais l'aire PDdM, lorsque la Lune est dans les syzygies, est le rectangle sous l'arc $P M$ et le rayon $P T$; et la somme de toutes les aires égales à celle-là, décrites pendant le temps que la Lune parcourt le cercle, est le rectangle de toute la circonférence et du rayon; et ce rectangle étant égal à deux cercles, est double du rectangle précédent. Donc les nœuds, avec une vitesse continuée uniformément et égale à celle qu'ils ont dans les syzygies lunaires, décriraient un espace double de celui qu'ils décrivent réellement ; et par conséquent le mouvement médiocre, qui étant continué uniformément ferait décrire aux nœuds l'espace qu'ils parcourent réellement d'un mouvement inégal, est la moitié du mouvement qu'ils ont dans les syzygies lunaires. Et comme le plus grand mouvement horaire, lorsque les nœuds sont dans les quadratures, est de $33^{\prime \prime} 10^{\prime \prime \prime} 33^{\text {iv }} 12^{\mathrm{v}}$, le mouvement médiocre horaire sera dans ce cas de $16^{\prime \prime} 35^{\prime \prime \prime} 16^{\text {iv }} 36^{\mathrm{v}}$. Or le mouvement horaire des nœuds étant toujours comme $A Z^{2}$ et l'aire $P D d M$ conjointement, il est encore dans les syzygies comme $A Z^{2}$ et l'aire $P D d M$ conjointement, ou, ce qui revient au même, comme $A Z^{2}$ (à cause qu'alors l'aire $P D d M$ est donnée) ; le mouvement médiocre sera aussi comme $A Z^{2}$, donc ce mouvement, lorsque les nœuds seront hors des quadratures, sera à $16^{\prime \prime} 35^{\prime \prime \prime} 16^{\text {iv }} 36^{\mathrm{v}}$ comme $A Z^{2}$ à $A T^{2}$. - C.Q.F.D.

\section{Proposition XXXI — Problème XII.}

Trouver le mouvement horaire des nœuds de la Lune dans un orbe elliptique.

(Fig. 8)

Que Qpmaq désigne une ellipse, $Q q$ son grand axe, $a b$ son petit axe, $Q A q B$ le cercle circonscrit ; $T$ la Terre placée au centre commun 
de l'ellipse et du cercle; $S$ le Soleil ; $p$ la Lune mue dans l'ellipse, et pm l'arc qu'elle décrit dans une particule donnée infiniment petite de temps ; $N n$ la ligne des nœuds ; $p K$ et $m k$ les perpendiculaires abaissées sur l'axe $Q q$ et prolongées jusqu'à ce qu'elles rencontrent le cercle en $P$ et en $M$, et la ligne des nœuds en $D$ et en $d$.

Cela posé, je dis que si la Lune décrit autour de la Terre des aires proportionnelles au temps, le mouvement horaire du nœud dans l'ellipse sera comme l'aire $p D d m$ et $A Z^{2}$ conjointement.

Pour le démontrer, soient menées $P F$ et $p f$ qui touchent en $P$ et $p$ le cercle et l'ellipse, qui rencontrent en $F$ et en $f$ la ligne des nœuds $T N$, et qui se rencontrent elles-mêmes ainsi que l'axe $T Q$ en $Y$. Soit pris $M L$ pour désigner l'espace que la Lune tournant dans le cercle, pourrait décrire d'un mouvement transversal par la force $3 I T$ ou $3 P K$, pendant qu'elle décrit l'arc $P M$. Et prenant $\mathrm{ml}$ pour l'espace que la Lune, tournant dans le même temps dans l'ellipse, décrirait par la même force $3 I T$ ou $3 P K$; enfin soient prolongées $L P$ et $l p$ jusqu'à ce qu'elles rencontrent le plan de l'écliptique en $G$ et en $g$; et soient tirées $F G$ et $f g$ dont la première $F G$ prolongée coupe $p f, p g$ et $T Q$ en $c$, $e$, et $R$, respectivement, et dont la seconde $f g$ prolongée coupe $T Q$ en $r$.

Il est clair que la force $3 I T$ ou $3 P K$ dans le cercle, étant à la force $3 I T$ ou $3 p K$ dans l'ellipse comme $P K$ à $p K$ ou comme $A T$ à $a T$; l'espace $M L$, décrit par la première force, sera à l'espace $m l$ décrit par la dernière, comme $P K$ à $p K$, c'est-à-dire, à cause des figures semblables PYKp et FYRc comme FR à $c R$. Mais, (par les triangles semblables $P L M, P G F$ ) $M L: F G=P L: P G$, c'est-à-dire, (à cause des parallèles $L k, P K, G R)=p l: p e$, ou, ce qui revient au même, (à cause des triangles semblables plm, pce $)=m l: c e$. Donc $L M: \operatorname{lm}$ ou $F R: c R=$ FG : ce.

De là il suit que si fg était à ce comme fy à $c Y$, ou comme fr à $c R$, c'est-à-dire, en raison composée de fr à $F R$ et de $F R$ à $c R$ ou de $f T$ à $F T$ et de $F G$ à $c e$, en ôtant de part et d'autre la raison de $F G$ à $c e$, il y aurait égalité entre la raison de $f g$ à $F G$ et celle de $f F$ à $F T$; c'est-àdire, que les angles à la Terre soutenus par $f g$ et $F G$, seraient égaux : ou, ce qui revient au même, les mouvements des nœuds dans l'ellipse et dans le cercle seraient égaux dans cette supposition, puisque ces angles seraient, parce que nous avons vu dans la Proposition précé- 
dente, les mouvements des nœuds dans le temps dans lequel la Lune parcourt l'arc $P M$ dans le cercle et l'arc $p m$ dans l'ellipse.

(Fig. 8)

Cela serait en effet ainsi, si fg était à $c e$ comme $f Y$ à $c Y$, c'est-àdire, si $f g$ était $=\frac{c e \times f Y}{c Y}$. Mais à cause des triangles semblables fgp, cep, on a $f g: c e=f p: c p$; donc $f g=\frac{c e \times f p}{c p}$; et par conséquent l'angle que $f g$ sous-tend réellement, est au premier angle que $F G$ soustend, c'est-à-dire, le mouvement des nœuds dans l'ellipse est au mouvement des nœuds dans le cercle comme cette ligne $f g$ ou $\frac{c e+f p}{c p}$ à la première valeur de $f g$ qu'on a trouvé $=\frac{c e \times f Y}{c Y}$, ou ce qui revient au même, en raison composée de $f p \times c Y$ à $f Y \times c p$, c'est-à-dire, en raison de $f p$ à $f Y$ et de $c Y$ à $c p$, ou bien encore, en menant $p h$ parallèle à $T N$ et rencontrant $F P$ en $h$, en raison composée de $F h$ à $F Y$ et de $F Y$ à $F P$; ou enfin dans la raison $F h$ à $F P$ qui est celle de $D p$ à $D P$, ou de l'aire Dpmd à l'aire DPMd.

Or comme, par le Cor. 1 de la Prop. 30 le mouvement horaire des nœuds dans le cercle est en raison composée de $A Z^{2}$ et de l'aire $D P M d$, le mouvement horaire des nœuds dans l'ellipse est donc en raison composée de l'aire Dpmd et de $A Z^{2}$. - C.Q.F.D.

Cor. C'est pourquoi, comme dans une position donnée des nœuds, la somme de toutes les aires $p D d m$ décrites pendant le temps que la Lune va d'une quadrature à un lieu quelconque $m$, est l'aire $m p Q E d$, terminée par la ligne $Q E$ tangente de l'ellipse ; et que la somme de toutes ces aires décrites dans une révolution entière est l'aire elliptique entière : le mouvement médiocre des nœuds dans l'ellipse sera au mouvement médiocre des nœuds dans le cercle, comme l'ellipse au cercle ; c'est-à-dire, $=T a: T A$ ou $=69: 70$. et par conséquent, puisque (Cor. 2 Proposition 30) le mouvement horaire médiocre des nœuds dans le cercle, est à $16^{\prime \prime} 35^{\prime \prime \prime} 16^{\text {iv }} 36^{\mathrm{v}}$ comme $A Z^{2}$ à $A T^{2}$, si on prend l'angle de $16^{\prime \prime} 21^{\prime \prime \prime} 3^{\text {iv }} 30^{\mathrm{v}}$, comme 69 à 70 , le mouvement horaire médiocre des nœuds dans l'ellipse sera à $16^{\prime \prime} 21^{\prime \prime \prime} 3^{\text {iv }} 30^{\mathrm{v}}$, comme $A Z^{2}$ à 
$A T^{2}$, c'est-à-dire, comme le carré du sinus de la distance du nœud au Soleil est au carré du rayon.

(Fig. 8)

Au reste, les aires que la Lune décrit autour de la Terre, étant parcourues plus promptement dans les syzygies que dans les quadratures, le temps doit diminuer dans les syzygies et augmenter dans les quadratures, et le mouvement des nœuds doit subir la même loi.

Or le moment de l'aire dans les quadratures de la Lune, est à son moment dans les syzygies comme 10973 à 11 073, et par conséquent, le moment médiocre dans les octants est à l'excès dans les syzygies et au défaut dans les quadratures, comme la demi-somme 11023 de ces nombres est à leur demi-différence 50. Ainsi à cause que le temps dans des parties égales de l'orbe de la Lune est réciproquement comme sa vitesse, le temps médiocre dans les octants sera à l'excès du temps dans les quadratures et à son défaut dans les syzygies, produit par cette cause, comme 11023 à 50 à peu près. Quant aux lieux placés entre les quadratures et les syzygies, je trouve que l'excès des moments de l'aire à chacun des lieux sur le plus petit moment dans les quadratures, est à peu près proportionnel au carré du sinus de la distance de la Lune aux quadratures; et par conséquent, la différence entre le moment dans un lieu quelconque, et le moment médiocre dans les octants, est comme la différence entre le carré du sinus de la distance de la Lune aux quadratures, et le carré du sinus de $45^{\circ}$ ou la moitié du carré du rayon; et l'incrément du temps dans chacun des lieux entre les octants et les quadratures, et son décrément entre les octants et les syzygies, sont dans la même raison.

Mais le mouvement des nœuds, pendant le temps que la Lune parcourt des parties égales d'orbe, est accéléré ou retardé en raison doublée du temps. Car ce mouvement, pendant que la Lune parcourt l'arc $P M$ (toutes choses d'ailleurs égales) est comme $M L$; et $M L$ est en raison doublée du temps. C'est pourquoi le mouvement des nœuds dans les syzygies, pendant le temps que la Lune parcourt des parties données de son orbe, est diminué dans la raison doublée du nombre 11073 au nombre 11023 ; et le décrément est au mouvement restant comme 100 à 10973 , et par conséquent au mouvement total à peu près comme 100 à 11073 . Or le décrément dans les lieux entre les octants et les syzygies et l'incrément entre les octants et les quadratu- 
res sont à peu près à ce décrément en raison composée de la raison du mouvement total dans ces lieux au mouvement total dans les syzygies, et de la raison que la différence entre le carré du sinus de la distance de la Lune à la quadrature, et la moitié du carré du rayon, a avec la moitié du carré du rayon.

Ainsi, si les nœuds sont dans les quadratures, et qu'on prenne deux lieux également distants de l'octant, et deux autres également distants de la syzygie et de la quadrature : ensuite, que des décréments des mouvements dans les deux lieux entre la syzygie et l'octant, on retranche les incréments des mouvements dans les deux autres lieux qui sont entre l'octant et la quadrature; le décrément restant sera égal au décrément dans la syzygie : ce dont il est facile de voir la raison. Delà il suit que le décrément médiocre qui doit être retranché du mouvement médiocre des nœuds, est la quatrième partie du décrément dans la syzygie.

Le mouvement total horaire des nœuds dans les syzygies, lorsque la Lune est supposée décrire des aires proportionnelles au temps autour de la Terre, a été trouvé précédemment de $32^{\prime \prime} 42^{\prime \prime \prime} 7^{\text {iv }}$; et le décrément du mouvement des nœuds, dans le temps que la Lune décrit plus promptement ce même espace, est suivant ce qu'on vient de dire, à ce mouvement, comme 100 à 11073 , donc ce décrément est de $17^{\prime \prime \prime}$ $43^{\text {iv }} 11^{\mathrm{v}}$ dont la quatrième partie $4^{\mathrm{m}} 25^{\text {iv }} 48^{\mathrm{v}}$ retranchée du mouvement horaire médiocre trouvé ci-dessus de $16^{\prime \prime} 21^{\prime \prime \prime} 3^{\text {iv }} 30^{\mathrm{v}}$ donne $16^{\prime \prime} 16^{\prime \prime \prime}$ $37^{\text {iv }} 42^{\mathrm{v}}$ pour le mouvement médiocre horaire corrigé.

Si les nœuds se trouvent hors des quadratures, et qu'on considère deux lieux également distants de part et d'autre des syzygies; la somme des mouvements des nœuds, lorsque la Lune sera dans ces lieux, sera à la somme des mouvements lorsque la Lune sera dans ces mêmes lieux, et que les nœuds seront dans les quadratures, comme $A Z^{2}$ à $A T^{2}$. Et les décréments des mouvements qui viennent des causes dont on a parlé, seront l'un à l'autre comme ces mouvements, c'est-à-dire, que les mouvements restants seront l'un à l'autre comme $A Z^{2}$ à $A T^{2}$, et les mouvements médiocres comme les mouvements restants. Donc le mouvement médiocre horaire corrigé, dans une position quelconque donnée des nœuds, sera à $16^{\prime \prime} 16^{\prime \prime \prime} 37^{\text {iv }} 42^{\mathrm{v}}$ comme $A Z^{2}$ à $A T^{2}$, c'est-à-dire, comme le carré du sinus de la distance des nœuds aux syzygies au carré du rayon. 


\section{Proposition XXXII. - Problème XIII.}

Trouver le mouvement moyen des nœuds de la Lune.

(Fig. 9)

Le mouvement moyen annuel est la somme de tous les mouvements médiocres horaires dans une année. Qu'on imagine un nœud allant vers $N$, et qu'on suppose de plus qu'à la fin de chaque heure il soit replacé dans son premier lieu; en sorte que malgré son mouvement propre, il conserve toujours la même position par rapport aux fixes. Qu'on suppose encore que pendant ce temps le Soleil, par le mouvement de la Terre, s'éloigne de ce nœud, et qu'il achève uniformément sa révolution annuelle apparente. Aa étant un très petit arc donné que la ligne TS menée au Soleil parcourt sur le cercle NAn dans un petit temps donné : le mouvement médiocre horaire sera, parce qu'on a fait voir ci-devant, comme $A Z^{2}$, c'est-à-dire, à cause des proportionnelles $A Z, Z Y$, comme le rectangle sous $A Z$ et $Z Y$ ou, ce qui revient au même, comme l'aire $A Z Y a$. Et la somme de tous les mouvements médiocres horaires depuis le commencement sera comme la somme de toutes les aires aYZA c'est-à-dire, comme l'aire NAZ. Or la plus grande aire $A Z Y a$ est égale au rectangle sous l'arc $A a$ et le rayon du cercle; et par conséquent, la somme de tous les rectangles dans le cercle entier sera à la somme d'autant de plus grands, comme l'aire de tout le cercle est au rectangle sous la circonférence entière et le rayon, c'est-à-dire, comme 1 à 2. Mais le mouvement horaire, répondant au grand rectangle, a été trouvé de $16^{\prime \prime} 16^{\prime \prime \prime} 37^{\text {iv }} 42^{\mathrm{v}}$, qui devient de $39^{\circ}$ $38^{\prime} 7^{\prime \prime} 50^{\prime \prime \prime}$ dans une année entière sidérale de 365 jours $6^{\mathrm{h}} 9^{\prime}$ : donc la moitié $19^{\circ} 49^{\prime} 3^{\prime \prime} \quad 55^{\prime \prime \prime}$ de ce mouvement est le mouvement moyen des nœuds qui répond à tout le cercle. Et le mouvement des nœuds, pendant que le Soleil va de $N$ en $A$, est à $19^{\circ} 49^{\prime} 3^{\prime \prime} 55^{\prime \prime \prime}$ comme l'aire $N A Z$ à tout le cercle.

Cela serait ainsi dans la supposition que le nœud fut remis à chaque heure à son premier lieu, et que le Soleil au bout d'une année retournât au même nœud d'où il était parti au commencement. Mais comme le mouvement du nœud est cause que le Soleil y revient plus tôt, il faut compter de combien le temps de ce retour est abrégé. 
Le Soleil parcourant par an $360^{\circ}$, et le nœud par son plus grand mouvement faisant dans le même temps $39^{\circ} 38^{\prime} 7^{\prime \prime}$ ' $50^{\prime \prime \prime}$ ou 39,6355 degrés ; et le mouvement médiocre de ce nœud dans un lieu quelconque $N$ étant à son mouvement médiocre dans ses quadratures, comme $A Z^{2}$ à $A T^{2}$, le mouvement du Soleil sera au mouvement du nœud au lieu $N$ comme $360 A T^{2}$ à $39,6355 A Z^{2}$, c'est-à-dire, comme $9,0827646 A T^{2}$ à $A Z^{2}$. Ainsi en supposant que toute la circonférence du cercle $N A n$ soit divisée en petites parties égales $A a$, le temps pendant lequel le Soleil parcourrait la petite partie $A a$, si le cercle était en repos, sera au temps pendant lequel il parcourra la même petite partie, ce cercle et les nœuds révolvants autour du centre $\mathrm{T}$, réciproquement comme $9,0827646 A T^{2}$ à $9,0827646 A T^{2}+A Z^{2}$. Car le temps est réciproquement comme la vitesse avec laquelle cette petite partie est parcourue, et cette vitesse est la somme des vitesses du Soleil et du nœud. Donc si le temps pendant lequel le Soleil parcourrait l'arc NA, indépendamment du mouvement du nœud, est représenté par le secteur NTA, et la petite partie de temps pendant laquelle il parcourrait un très petit arc $A a$ par la petite portion $A T a$ de ce secteur; que l'on abaisse $a Y$ perpendiculaire sur $N n$, et qu'on prenne $d Z$ sur $A Z$ d'une longueur telle que le rectangle $d Z \times Z Y$ soit à la petite portion $A T a \mathrm{du}$ secteur comme $A Z^{2}$ à 9,0827646 $A T^{2}+A Z^{2}$, c'est-à-dire, en sorte que $d Z: \frac{1}{2} A Z=A T^{2}: 9,0827646 A T^{2}+A Z^{2}$; le rectangle $d Z \times Z Y$ représentera le décrément du temps causé par le mouvement du nœud, pendant le temps total pendant lequel l'arc $A a$ a été parcouru. Et si la courbe $N d G n$ est le lieu des points $d$, l'aire curviligne $N d Z$ sera le décrément total pendant le temps employé à parcourir l'arc $N A$ entier, et par conséquent l'excès du secteur $N A T$ sur l'aire $N d Z$ sera ce temps total. Or comme le mouvement du nœud dans un temps plus court est moindre dans la raison du temps, l'aire $A a Z Y$ devra être diminuée dans la même raison; ce qui se fera en prenant sur $A Z$ l'intervalle $e Z$ qui soit à la ligne $A Z$ comme $A Z^{2}$ à $9,0827646 A T^{2}+A Z^{2}$. Par ce moyen le rectangle $e Z \times Z Y$ sera à l'aire $A Z Y a$ comme le décrément du temps employé à parcourir l'arc $A a$, au temps total dans lequel il serait parcouru si le nœud était en repos, et par conséquent ce rectangle répondra au décrément du mouvement du nœud. Et si la courbe $\mathrm{NeFn}$ est le lieu des points $e$, l'aire totale $\mathrm{NeZ}$, qui est la somme de tous les décréments, répondra au décrément total, pendant le temps employé à parcourir l'arc $A N$, et l'aire restante $N A e$ répondra au mou- 
vement restant, qui est le vrai mouvement du nœud, pendant le temps pendant lequel l'arc total NA est parcouru par les mouvements réunis du Soleil et des nœuds.

Mais en employant les méthodes des suites infinies, on trouve que l'aire du demi-cercle est à l'aire de la figure $N e F n$ cherchée, environ comme 793 à 60 . Donc, comme le mouvement qui répondait au cercle entier était de $19^{\circ} 49^{\prime} 3^{\prime \prime} 55^{\prime \prime \prime}$ le mouvement qui répond au double de la figure $\mathrm{NeFn}$ sera de $1^{\circ} 29^{\prime} 58^{\prime \prime} 2^{\prime \prime \prime}$ qui, étant soustrait du premier mouvement, donnera $18^{\circ} 19^{\prime} 5^{\prime \prime} 5^{\prime \prime \prime}$ pour le mouvement total du nœud par rapport aux fixes entre ses propres conjonctions avec le Soleil ; retranchant ensuite ce mouvement du mouvement annuel du Soleil qui est de $360^{\circ}$, on aura $341^{\circ} 40^{\prime} 54^{\prime \prime} 7^{\prime \prime \prime}$ pour le mouvement du Soleil entre ces mêmes conjonctions. Et ce mouvement est au mouvement annuel de $360^{\circ}$, comme le mouvement du nœud ci-devant trouvé de $18^{\circ} 19^{\prime} 5^{\prime \prime} 53^{\prime \prime \prime}$ à son mouvement annuel, qui par conséquent sera de $19^{\circ} 18^{\prime} 1^{\prime \prime} \quad 23^{\prime \prime \prime}$. Et c'est là le mouvement moyen des nœuds dans une année sidérale. Ce mouvement, par les tables astronomiques, est de $19^{\circ} 21^{\prime} 21^{\prime \prime} 50^{\prime \prime \prime}$. Ainsi la différence est moindre que $\frac{1}{100}$ partie du mouvement total, et elle vient vraisemblablement de l'excentricité de l'orbe de la Lune, et de son inclinaison au plan de l'écliptique. Par l'excentricité de cet orbe le mouvement des nœuds est un peu trop accéléré, et son inclinaison le retarde un peu trop, ce qui le réduit à peu près à sa juste quantité.

\section{Proposition XXXIII. - ProblèMe XIV.}

Trouver le mouvement vrai des nouds de la Lune.

(Fig. 9)

Le temps étant représenté par l'aire $N T A-N d Z$ l'aire $N A e$ représente le mouvement vrai, ainsi il est donné par les quadratures. Comme le calcul serait pénible par cette méthode, il vaut mieux employer la construction suivante.

(Fig. 10)

Du centre $C$, et d'un intervalle quelconque $C D$, soit décrit le cercle $B E F D$, et soit prolongée $C D$ en $A$, en sorte que $A B$ soit à $A C$ comme le mouvement moyen à la moitié du mouvement vrai médiocre, lorsque les nœuds sont dans les quadratures, c'est-à-dire, comme $19^{\circ} 18^{\prime}$ 
$1^{\prime \prime} 23^{\prime \prime \prime}$ à $19^{\circ} 49^{\prime} 3^{\prime \prime} \quad 55^{\prime \prime \prime}$. BC sera par conséquent à $A C$ comme la différence $0^{\circ} 31^{\prime} 2^{\prime \prime} 32^{\prime \prime \prime}$ de ces mouvements au dernier mouvement de $19^{\circ} 49^{\prime} 3^{\prime \prime} \quad 55^{\prime \prime \prime}$, c'est-à-dire, comme 1 à $38 \frac{3}{10}$; soit ensuite tirée par le point $D$ la ligne indéfinie $G g$, qui touche le cercle en $D$; et soit pris l'angle $B C E$ ou $B C F$ égal au double de la distance du Soleil au lieu du nœud qui est trouvé par le mouvement moyen ; enfin soit tirée $A E$ ou $A F$ qui coupe la perpendiculaire $D G$ en $G$; et soit pris un angle qui soit au mouvement total du nœud entre ses syzygies (c'est-à-dire à $9^{\circ}$ $\left.11^{\prime} 3^{\prime \prime}\right)$ comme la tangente $D G$ à la circonférence entière du cercle $B E D$; cet angle (au lieu duquel on peut prendre l'angle $D A G$ ) étant ajouté au mouvement moyen des nœuds lorsqu'ils passent des quadratures aux syzygies, et étant soustrait de ce mouvement moyen lorsqu'ils passent des syzygies aux quadratures, on aura leur mouvement vrai. Car le résultat de cette opération s'accorde à très peu de choses près avec ce que l'on trouverait en exprimant le temps par l'aire NTA - NdZ et le mouvement du nœud par l'aire NAe : comme on peut s'en assurer par le calcul.

C'est là l'équation semestre du mouvement des nœuds. Il y a aussi une équation de ce mouvement pour chaque mois, mais elle n'est pas nécessaire pour trouver la latitude de la Lune. Car la variation de l'inclinaison de l'orbe de la Lune au plan de l'écliptique, éprouve une double inégalité, l'une tous les six mois, et l'autre tous les mois; cette inégalité de tous les mois et l'équation des nœuds pour chaque mois se compensent et se corrigent tellement l'une l'autre, qu'on peut les négliger en déterminant la latitude de la Lune.

Cor. Il est clair, par cette Proposition et par la précédente, que les nœuds sont stationnaires dans leurs syzygies, que dans leurs quadratures ils rétrogradent d'un mouvement horaire de $16^{\prime \prime} 19^{\prime \prime} 26^{\mathrm{iv}}$, et que $1^{\prime}$ 'équation du mouvement des nœuds dans les octants est de $1^{\circ} 30^{\prime}$, ce qui s'accorde très bien avec les phénomènes célestes.

\section{SCHOLIE.}

J. Machin professeur d'astronomie à Gresham et Henri Pemberton M. D. ont trouvé chacun de leur côté le mouvement des nœuds par une autre méthode que la précédente, et on a fait mention de cette autre méthode dans un autre lieu. Les écrits de l'un et de l'autre que j'ai 
vus, contenaient chacun deux Propositions et s'accordaient parfaitement. Je joindrai ici l'écrit du Docteur Machin parce qu'il m'est tombé plutôt entre les mains. 


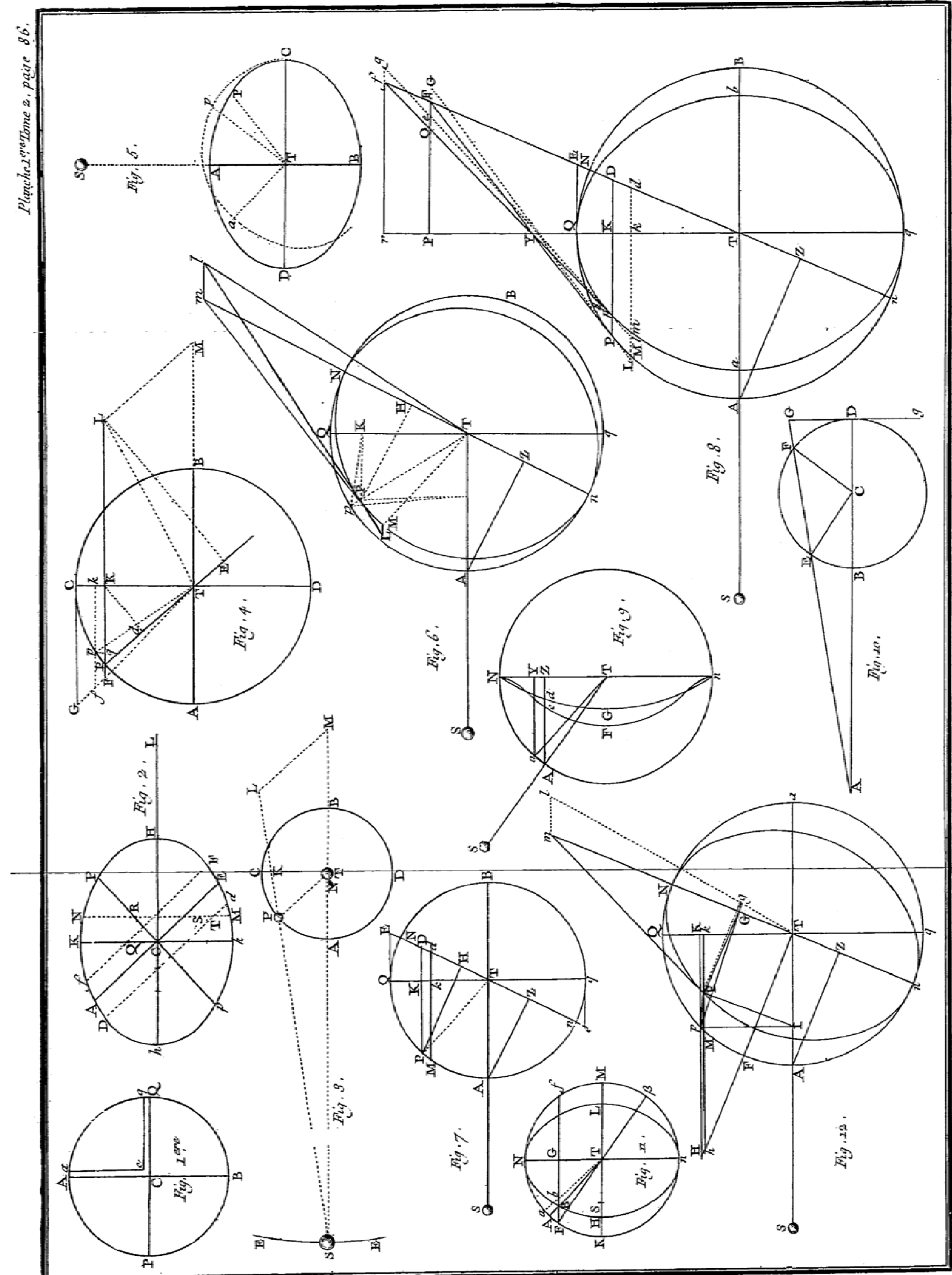

Planche I 